\title{
Understanding rapid charge and discharge in nano-structured lithium iron phosphate cathodes
}

\author{
M. Castle ${ }^{1,4}$, G. Richardson ${ }^{2,3}$, and J. M. Foster ${ }^{1,3,5}$ \\ ${ }^{1}$ School and Mathematics and Physics, University of Portsmouth, Lion \\ Terrace, PO1 3HF, UK \\ ${ }^{2}$ Mathematical Sciences, University of Southampton, University Rd., SO17 \\ 1BJ, UK \\ ${ }^{3}$ The Faraday Institution, Quad One, Becquerel Avenue, Harwell Campus, \\ Didcot, OX11 0RA, UK

January 23, 2021

\begin{abstract}
A Doyle-Fuller-Newman (DFN) model for the charge and discharge of nano-structured lithium iron phosphate (LFP) cathodes is formulated on the basis that lithium transport within the nanoscale LFP electrode particles is much faster than cell discharge, and is therefore not rate limiting. We present some numerical solutions to the model and show that for relevant parameter values, and a variety of C-rates, it is possible for sharp discharge fronts to form and intrude into the electrode from its outer edge(s). These discharge fronts separate regions of fully utilised LFP electrode particles from those that are not. Motivated by this observation an asymptotic solution to the model is sought. The results of the asymptotic analysis of the DFN model lead to a reduced order model, which we term the reaction front model (or RFM). Favourable agreement is shown between solutions to the RFM and the full DFN model in appropriate parameter regimes. The RFM is significantly cheaper to solve than the DFN model and therefore has the potential to be used in scenarios where computational costs are prohibitive, e.g. in optimisation and parameter estimation problems or in engineering control systems.
\end{abstract}

\section{Introduction}

Lithium iron phosphate (LFP) has been developed as a cathode material for lithium-ion batteries [37]. Despite having a slightly lower energy density than nickel-manganese-cobalt 
oxide (which is the most widely used cathode material today), its high power density (i.e. the ability to extract energy quickly), low cost, and safety make LFP an environmentally friendly alternative to the cobalt-based electrodes. LFP is used in a range of commercial applications, including electrical vehicles, backup home generators, consumer electronics, and marine technologies, and Tesla plans to deploy LFP-based cathodes in their forthcoming home storage batteries [4,52]. Initially the use of LFP in lithium-ion batteries was hampered by its low electrical conductivity which hindered the flow of electronic current into the cathode. However, the electronic conductivity of the network between LFP electrode particles has been improved by adding carbon black [14, 22, 24] and the conductivity of the LFP particles increased by doping (e.g. Wang et al. [54]). LFP is a phase separating material, as evidenced by its very flat open circuit voltage (OCV) curve (the potential is almost independent of degree of discharge until the particle is fully discharged [37]). Thus, unlike electrode materials with non-flat OCVs, electrode particles within the electrode frequently discharge at different rates which depend sensitively on the solid state electric potential within the electrode and electrolyte. The insertion/extraction of lithium within the electrode can therefore be highly non-uniform during operation. There is thus scope for significant improvements to be made to LFP cathodes by carefully tuning the electrical properties of the electrolyte and the electrode conduction network, a fact that has been noted previously by Johns et al. [25] who demonstrate that changes to the electrolyte properties in a LFP cathode can result in increases in maximum discharge rate of around two orders of magnitude. Volumetric energy density and rate capability can also be improved by using a blend of differently sized electrode particles $[27,28,47]$. Liu et al. [28] showed that by using a nano-micro structured LFP electrode it is possible to achieve higher compaction density, lower resistance, superior rate capability, and higher volumetric energy density whilst still maintaining excellent cycling stability [28].

Diffusion of lithium within LFP particles is highly anisotropic occurring preferentially along channels in the $b$-direction of the crystal [31]. In nanoscale crystals this diffusion is observed to occur extremely rapidly. Kang and Cedar [7], for example, have shown that, with the application of a glassy lithium phosphate surface coating to enable lithium transport around the surface of the particle, $50 \mathrm{~nm}$ particles can be discharged in around 20s. However lithium diffusion within larger (micro-)particles is known to be much slower and more isotropic. This has been explained by blockage of the preferred $b$-direction channels by defects and grain boundaries; this forces lithium transport along tortuous paths with components in the high-resistance $a$ - and $c$-directions [31]. For high power applications LFP nanoparticles are hence to be preferred and, where they are used in batteries, diffusion within the LFP particles is so fast that its effects can be neglected in modelling battery charge and discharge. The limiting factors in the performance of such cathodes are lithium ion transport and electrical conduction within the electrolyte and solid state electrical conduction between electrode particles. This observation motivates our modelling approach to the problem.

The standard framework for modelling charge transport in lithium ion batteries has been set out in a series of works by Doyle, Fuller and Newman (i.e. in $[13,19])$; henceforth referred to as the DFN modelling framework. This comprises one-dimensional (through the thickness of the electrode) descriptions of (i) electrical conduction between electrode particles via a porous network of polymer binder and conducting additive and (ii) lithium ion transport in the electrolyte. These couple to a diffusion model for lithium transport within 
representative electrode particles (at each location through the electrode width) via ButlerVolmer conditions for charge transfer (via lithium (de-)intercalation reactions) between the electrode and the electrolyte, on the surfaces of the electrode particles. This framework is adopted here but with the simplifying assumption that lithium transport within the electrode particles is extremely rapid relative to the timescale of (dis)charge of the device. The upshot is that the diffusion equations for transport in the electrode particles can be replaced with ODEs. We that DFN models which were originally postulated on an ad-hoc basis have since been derived as the limit of microscopic (particle sized) description of the battery using systematic asymptotic homogenisation techniques $[8,44]$.

Previous modelling studies of LFP half-cells using the DFN framework include work by Srinivasan and Newman [49] and Dargaville and Farrell [10, 11]. Both [49] and [10] adopt a "shrinking core" model to describe lithium transport and phase change within the electrode particles. In both these works comparison was made to experimental discharge curves measured from a half cell cathode with micron sized LFP electrode particles in [49]. Whilst agreement to the experimental data in the former [49] is unexceptional it is greatly improved by allowing for two sizes of electrode particle. In the latter [10] agreement to the experiment is reasonable but the theory requires an extra layer of modelling to account for electrode particle clustering. The "shrinking core" model for LFP has now largely been discredited [9] as a result of a better understanding of the lithium transport processes within this material [31]; [9] notes that the agreement obtained between the data presented in [49] and "shrinking core" based models can be explained by the large electrical resistance within the electrode limiting the discharge, rather than by lithium transport within the electrode particles. More recent work by Dargaville and Farrell [11] uses a phase field description of lithium transport within the electrode particles and, perhaps surprisingly, finds poor agreement with the experimental data of Zheng et al. [56]. Here, we avoid having to confront questions about the details of how lithium transport occurs within LFP by restricting our interest to nanoparticles.

The work of Bai et al. suggests that, during high-rate discharge, phase-separation dynamics can be neglected; these effects are dynamically suppressed during high-current operation due to the fact that the surface over-potential can easily exceed the solid-solution voltage barrier in smaller particles and thus removes the thermodynamic driving force for phase separation once the current becomes comparable to the exchange current. On this basis they conclude that it is only for large particles and low currents that phase separation plays a major role.

Bazant and co-workers have been proponents of the use of the Cahn-Hilliard equation in describing (de)lithiation dynamics of phase separating materials [3]. They introduced free energy models for LFP (two phases with coherent nucleation) and graphite (three phases, neglecting nucleation) to predict experimental data [18]. Particularly relevant to the work here is that they have shown that discharge fronts can exist in LFP electrodes. Moreover, there is a propensity for sharper reaction fronts in electrodes where there is little variation in the electrode particle size. This is attributed to how the numbers of grain boundaries are likely to vary with particle size and so smaller particles (with fewer grain boundaries) are likely to react faster than larger ones causing a more diffuse reaction front.

Johns et al. [25] have performed experiments on nano-structured LFP half-cells in order to highlight the importance of the electrolyte in discharge performance. In particular they 
show that by increasing the diffusivity of the electrolyte they were able to discharge at much faster rates. They also compare their results to a sharp discharge front (SDF) model in which an interface propagates into the half-cell from the separator; behind this front the electrode material is fully discharged (i.e. fully lithiated) and in front of it is fully charged (i.e. fully de-lithiated). While such a model could be criticised for being overly crude its premise is, to a large extent, borne out by the results of the DFN-type model presented in this work which also predicts discharge fronts.

Here, we investigate the (dis)charge of a nano-structured electrode, such as that considered by Owen et al. [25], using a DFN model at moderate to aggressive rates. We show that whilst the SDF model presented by Owen et al. encapsulates some of the key features of the (dis)charge, physical properties such as the ionic conductivity and diffusivity of the electrolyte, which were assumed to be constant in their SDF model, significantly affect cell discharge. Here we apply formal asymptotic methods to the full DFN model in order to systematically derive a reduced order model for the charge/discharge of a nano-structured LFP half-cell cathode. This reduced order model is capable of predicting the ionic and electronic potentials throughout discharge and gives a precise method for predicting the termination of cell (dis)charge. As we will show, the physics retained by our systematic derivation, which is not present in the simplistic SDF model presented by Johns et al. [25], is crucial in explaining some features of LFP electrode behaviour. In this context we note that other reduced order models for lithium ion battery charge and discharge, appropriate for other battery chemistries, have been derived directly from the DFN model, see for example [32, 45].

The remainder of this work is structured as follows. In $\S 2$ we describe the DFN model for a LFP half-cell with the simplification that transport within the electrode particles is rapid, provide a suitable non-dimensionalisation of the model, and discuss typical values for these resulting dimensionless parameters. In the subsequent section, §3, we present some numerical solutions to the model for relevant values of the parameters. We show that it is possible for the solution to exhibit sharp discharge fronts which separate utilised regions of the electrode from regions that are still undergoing reaction. In $\S 4$, we systematically derive an asymptotic reduction to the model in a distinguished limit in which the dimensionless reaction rate constant is large so that in significant portions of the electrode, the electrolyte and electrode particles are almost in equilibrium with one another. In $\S 6$ we validate the reduction by comparing it to numerical simulations of the full DFN model. Finally in $\S 7$ we present our conclusions.

\section{Problem formulation}

We consider a one-dimensional porous LFP-based half-cell cathode, in $x^{*} \in(0, L)$, sandwiched between a current collector located on $x^{*}=L$ and a porous separator in $x^{*} \in\left(-L_{s}, 0\right)$. In $x^{*}<-L_{s}$ is a Li metal counter electrode. This model geometry is illustrated in figure 1. The electrochemical behaviour of this device is described by the DFN model [13, 19, 44]. 
Macroscopic transport The ionic transport and current flow through the electrolyte, in both the porous electrode and binder, is described in $x^{*} \in\left(-L_{s}, L\right)$ by the PDEs

$$
\begin{gathered}
\epsilon_{l} \frac{\partial c^{*}}{\partial t^{*}}+\frac{\partial \mathcal{F}^{*}}{\partial x^{*}}=0, \quad \mathcal{F}^{*}=-\mathcal{B}^{*}\left(x^{*}\right) D^{*}\left(c^{*}\right) \frac{\partial c^{*}}{\partial x^{*}}-\left(1-t^{+}\right) \frac{j^{*}}{F} \\
\frac{\partial j^{*}}{\partial x^{*}}=F b_{c} G^{*}, \quad j^{*}=-\mathcal{B}^{*}\left(x^{*}\right) \kappa^{*}\left(c^{*}\right)\left(\frac{\partial \phi^{*}}{\partial x^{*}}-\frac{2 R_{g} T}{F} \frac{1-t^{+}}{c^{*}} \frac{\partial c^{*}}{\partial x^{*}}\right)
\end{gathered}
$$

Here $t^{*}$ denotes time, $\epsilon_{l}$ is the local volume fraction of electrolyte, $c^{*}$ is the molar ion concentration in the electrolyte, $\mathcal{F}^{*}$ is the effective flux of the negative counter-ions across the electrolyte, $D^{*}$ is the ionic diffusivity of electrolyte, $\mathcal{B}^{*}$ is the permeability factor, $t^{+}$is the transference number, $j^{*}$ is the ionic current density in the electrolyte, $F$ is Faraday's constant, $b_{c}$ is the BET surface area (the surface area of electrode particles per unit volume of cathode), $G^{*}$ is the Butler-Volmer (BV) reaction rate per unit area (i.e. the flux of Li-ions per unit area through the surface of the electrode particles), $\kappa^{*}$ is the ionic conductivity, $\phi^{*}$ is the electric potential in the electrolyte, $R_{g}$ is the (molar/universal/ideal) gas constant, and $T$ is absolute temperature. The dependencies of $D^{*}$ and $\kappa^{*}$ on $c^{*}$ are shown in figure 2. Here we formulate (1) in terms of the negatively charged counterion flux, rather than the $\mathrm{Li}^{+}$ion flux, because these ions are not (de)intercalated into the electrode particles and are thus globally conserved throughout the electrolyte. It follows that (1) has strictly conservative form and is thus more easily solved numerically. This is contrast to the conservation equation for $\mathrm{Li}^{+}$ions which contains source/sink terms accounting for the (de)intercalation process. The current flow through the solid matrix (comprised of electrode particles, binder and conductive additives), situated in $x^{*} \in(0, L)$, satisfies

$$
\frac{\partial j_{c}^{*}}{\partial x^{*}}=-F b_{c} G^{*}, \quad j_{c}^{*}=-\sigma_{c} \frac{\partial \phi_{c}^{*}}{\partial x^{*}} .
$$

where $\sigma_{c}$ is the electronic conductivity and $\phi_{c}^{*}$ and $j_{c}^{*}$ are the electric potential and the electronic current density in the solid matrix, respectively. The charge transport equations in the electrolyte (1)-(3) satisfy the following boundary conditions on $x=-L_{s}$, the separator edge in contact with the metallic lithium electrode,

$$
\left.j^{*}\right|_{x^{*}=-L_{s}}=\frac{I^{*}\left(t^{*}\right)}{A},\left.\quad \mathcal{F}^{*}\right|_{x^{*}=-L_{s}}=0,\left.\quad \phi^{*}\right|_{x^{*}=-L_{s}}=0
$$

where $I^{*}\left(t^{*}\right)$ is the current in the external circuit and $A$ is the cross-sectional area of the half-cell. These conditions demand that current enters the half-cell through the separator as ionic current, that there is no flux of the negative counter-ions on the metallic lithium electrode, and that potential is measured with respect to the metallic lithium electrode. On the current collector (1)-(3) satisfy the conditions

$$
\left.j_{c}^{*}\right|_{x^{*}=L}=\frac{I^{*}\left(t^{*}\right)}{A},\left.\quad \mathcal{F}^{*}\right|_{x^{*}=L}=0,\left.\quad j^{*}\right|_{x^{*}=L}=0,
$$

requiring that current flows from the half-cell into the current collector as electronic current and that there is no current flow or ion flux into the current collector. 


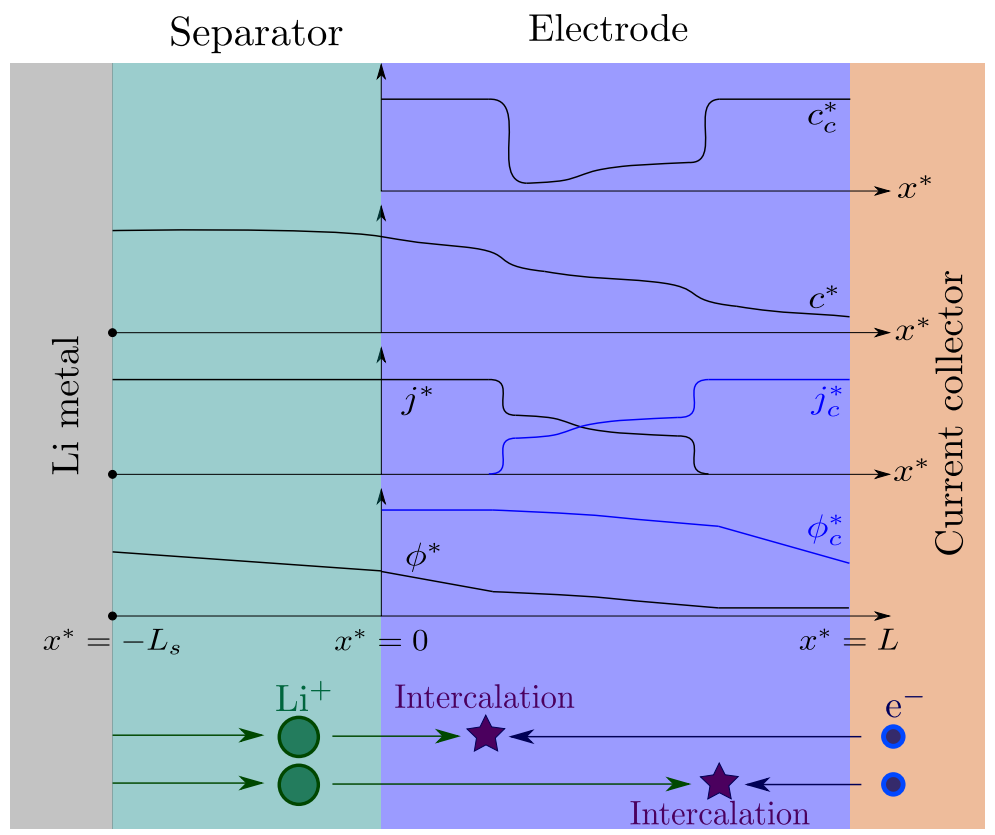

Figure 1: Diagram showing dynamics of a LFP half-cell.

Equations on the microstructure In order that the (de)intercalation reaction rates can be evaluated, the surface concentration on the electrode particles must be determined. It is assumed that the C-rate (i.e. the current drawn from the cell measured in units of the current needed to discharge the cell completely in 1 hour) is large enough that mosaic effects found in phase-separating electrode materials such as LFP can be neglected [29]. This necessitates solving microscopic transport problems within representative electrode particles through the thickness of the electrode. Since we assume the electrode is formed from nanoparticulate LFP the lithium concentration is, to a good approximation, spatially uniform within each particle. This can be justified by noting that the timescale for transport in the solid $\tau_{c}=R^{2} / D_{c}$, where $R$ is the electrode particle radius and $D_{c}$ is the typical diffusivity of lithium in LFP, is very short. Taking $R=300 \mathrm{~nm}[12,33,56]$ and $D_{c}=9 \times 10^{-14} \mathrm{~m} \mathrm{~s}^{-2}[26]$ we find that $\tau_{c}=1 \mathrm{~s}$, and so provided we do not approach rates of 3,600C it is reasonable to say that $\tau_{c} \ll \tau$, where $\tau$ is the timescale for cell discharge, and hence the electrode particles are quasi-steady on the timescale of cell discharge. Thus, the transport problem inside the (spherical) LFP nanoparticles is approximated by

$$
\frac{d c_{c}^{*}}{d t^{*}}=-\frac{3 G^{*}}{R}
$$

In the forthcoming sections we show that solutions to the model can exhibit behaviour in which the lithium intercalation occurs primarily within narrow reaction regions that propagate through the electrode. In such scenarios, the characteristic timescale for (de)lithiation of a particle is reduced to $\tau W / L$ where $W$ is the width of the region in which the reaction is concentrated. Hence, the caveat on the validity approximation (6) of the transport problem inside the LFP electrode particles $\left(\tau_{c} \ll \tau\right)$ should be amended to be given by $\tau_{c} \ll \tau W / L$. As we shall see later, in (21) and (64), a typical range of values for the ratio $W / L$ is between 
$10^{-2}$ and 1 , and as such the approximation (6) is often justified provided we do not approach rates of between $36 \mathrm{C}$ and $3,600 \mathrm{C}$ (depending on the device architecture).

The Butler-Volmer reaction rate The flux $G^{*}$ of Li-ions across the surface of an electrode particle, into the electrolyte, is modelled by the Butler-Volmer (BV) reaction rate $[5]$,

$$
G^{*}= \begin{cases}0, & -L_{s}<x^{*}<0 \\ k_{0} c^{* 1 / 2} c_{c}^{* 1 / 2}\left(\hat{c}_{c}-c_{c}^{*}\right)^{1 / 2}\left(\exp \left(\frac{F \eta^{*}}{2 R_{g} T}\right)-\exp \left(-\frac{F \eta^{*}}{2 R_{g} T}\right)\right), & 0 \leq x^{*}<L\end{cases}
$$

where $c_{c}^{*}$ is the maximum lithium ion concentration of the active material, $k_{0}$ is a reaction constant and $\eta^{*}$, the over-potential, is defined by

$$
\eta^{*}=\phi_{c}^{*}-\phi^{*}-U_{c}^{*}\left(c_{c}^{*}\right)
$$

Here $U_{c}^{*}\left(c_{c}^{*}\right)$, the equilibrium potential of the cathode electrode material (cf figure 2), is an experimentally measured function of the intercalated Li-ion concentration $c_{c}^{*}$ on the surface of the particle.

The half-cell potential The voltage drop across the device, $V^{*}\left(t^{*}\right)$, is comprised of two parts: the potential difference between the electrode at the current collector and separator; and the Ohmic drop across the current collector interface caused by contact resistance, $R_{c}$. On recalling the boundary condition (4c) an expression for the half-cell voltage is given by

$$
V^{*}\left(t^{*}\right)=\left.\phi_{c}^{*}\right|_{x^{*}=L}-R_{c} I^{*}(t)
$$

Geometry of the electrode The electrode is assumed to be locally periodic and comprised of identically-sized spherical electrode particles so that $n$, the particle number density, and $\epsilon_{p}$, the electrode particle volume fraction, are given by

$$
n=\frac{b_{c}}{4 \pi R^{2}}, \quad \epsilon_{p}=\frac{4}{3} \pi R^{3} n .
$$

It follows that $b_{c}$, the BET surface area, is related to the volume fraction and radius of particles via

$$
b_{c}=\frac{3 \epsilon_{p}}{R} .
$$

Initial conditions The problem is closed by specifying initial conditions on the lithium concentrations in the electrolyte and in the electrode particles. Here, we specify initial conditions corresponding to an electrode that has been allowed to reach equilibrium, so that the lithium concentrations in the electrolyte and the electrode particles are spatially uniform, and write

$$
\left.c^{*}\right|_{t^{*}=0}=\hat{c},\left.\quad c_{c}^{*}\right|_{t^{*}=0}=\alpha \hat{c}_{c} .
$$

Here $\alpha$ specifies the initial state of charge of the half-cell ( $\alpha=0$ corresponds to fully charged and $\alpha=1$ to fully discharged electrode particles). 
Table 1: Typical parameter values for a LFP half-cell.

\begin{tabular}{|c|c|c|}
\hline Parameter, symbol [unit] & Value & Reference \\
\hline \multicolumn{3}{|l|}{ Electrolyte Parameters } \\
\hline Electrode thickness, $L[\mu m]$ & 60 & {$[12,16,25,33,49,56]$} \\
\hline Separator thickness, $L_{s}[\mu m]$ & 25 & {$[40,49,51,55,57]$} \\
\hline Volume fraction of electrode particles, $\epsilon_{p}$ & 0.437 & [33] \\
\hline Volume fraction / porosity of electrolyte, $\epsilon_{l}$ & 0.463 & {$[33,38]$} \\
\hline Initial salt concentration, $\hat{c}\left[\mathrm{~mol} \mathrm{~m}^{-3}\right]$ & 1000 & {$[48]$} \\
\hline Transference number, $t^{+}$ & 0.38 & {$[50,53]$} \\
\hline \multicolumn{3}{|l|}{ Electrode Parameters } \\
\hline Reaction rate constant, $k_{0}\left[\mathrm{~mol}^{-0.5} \mathrm{~m}^{3.5} \mathrm{~s}^{-1}\right]$ & $1 \times 10^{-10}$ & {$[26]$} \\
\hline Particle radius, $R$ [nm] & 300 & {$[12,33,56]$} \\
\hline Matrix conductivity of modern electrodes, $\sigma_{c}\left[S \mathrm{~m}^{-1}\right]$ & 3.49 & {$[15]$} \\
\hline Matrix conductivity of older electrodes, $\sigma_{c}\left[S \mathrm{~m}^{-1}\right]$ & 0.005 & {$[34,48]$} \\
\hline Maximum concentration in the solid, $\hat{c}_{c}\left[\mathrm{~mol} \mathrm{~m} \mathrm{~m}^{-3}\right]$ & 22806 & {$[26]$} \\
\hline $\begin{array}{c}\text { Initial state of charge, } \alpha \\
\text { Other Parameters }\end{array}$ & 0.035 & {$[40]$} \\
\hline Faraday constant, $\mathrm{F}\left[\mathrm{Cmol}^{-1}\right]$ & 96487 & {$[49]$} \\
\hline Universal gas constant, $R_{g}\left[\mathrm{Jmol}^{-1} \mathrm{~K}^{-1}\right]$ & 8.3144 & {$[17]$} \\
\hline Temperature, $T[K]$ & 298 & {$[17]$} \\
\hline Cross-sectional area of electrode, $A\left[\mathrm{~m}^{2}\right]$ & $1 \times 10^{-4}$ & {$[33,46,48]$} \\
\hline Contact resistance, $R_{c}[\Omega]$ & $3.58 \times 10^{-3}$ & [10] \\
\hline
\end{tabular}

State of charge The state of charge of the cell is taken to be the total average concentration of lithium in the electrode particles normalised with respect to the theoretical capacity. The state of charge is denoted by, $\left\langle c_{c}\right\rangle$, and is given by

$$
\left\langle c_{c}\right\rangle=\int_{x^{*}=0}^{x^{*}=L} \frac{c_{c}^{*}}{\hat{c}_{c}} d x^{*},
$$

and as such, it follows from (12), that $\left.\left\langle c_{c}\right\rangle\right|_{t^{*}=0}=\alpha$.

C-rate The C-rate is a dimensionless measure of the (dis)charge rate. For a cathode undergoing galvanostatic (dis)charge we define the C-rate as the current draw/supply divided by the theoretical capacity of the cathode. However, it is worth pointing out that some authors choose to normalise with respect to an experimentally measured capacity rather than the theoretical one. Nonetheless a common definition, and the one we shall adopt is

$$
\text { C-rate }=\frac{3600 I^{*}}{L A \epsilon_{p} F \hat{c}_{c}} .
$$

Thus, provided the full capacity is accessible, a C-rate of 1 will bring the cathode from fully charged to discharged in 1 hour. 
(a)

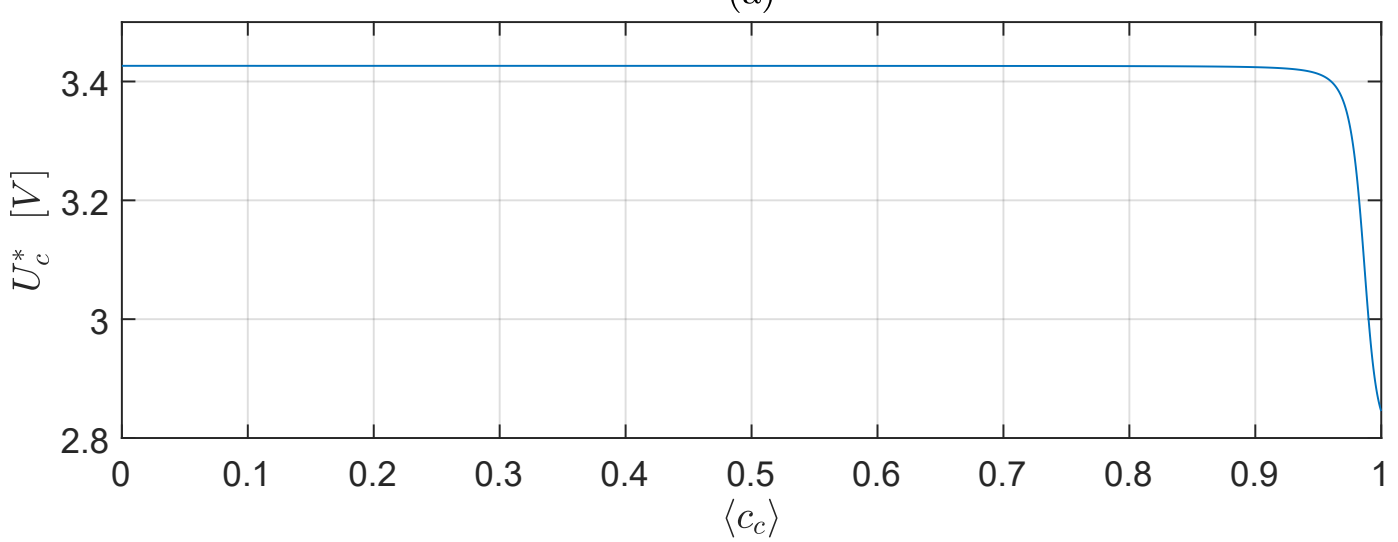

(b)

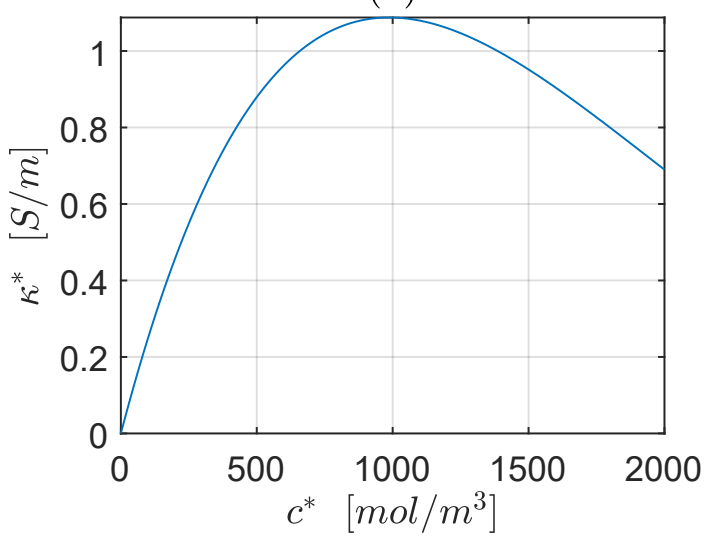

(c)

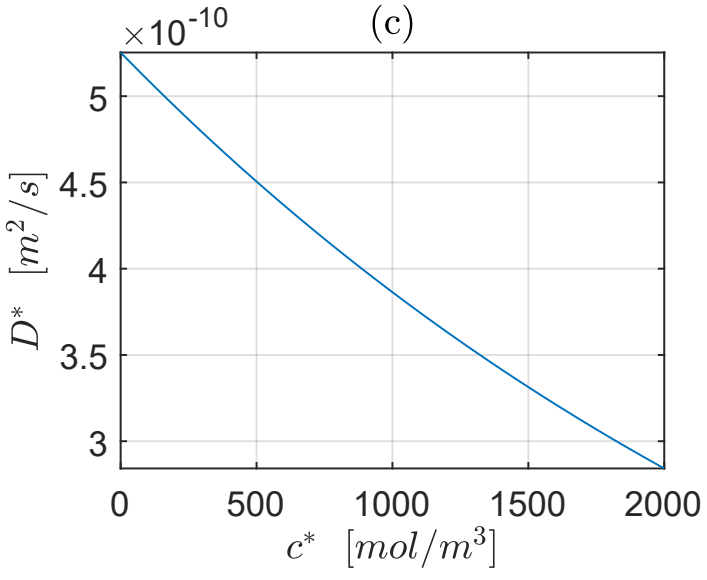

Figure 2: Panel (a) shows the equilibrium potential for LFP. Panels (b) and (c) show the ionic conductivity and diffusivity of lithium in the electrolyte respectively. 


\section{$2.1 \quad$ Non-dimensionalisation}

In order to conduct an asymptotic analysis of the problem the model (1)-(13) is first nondimensionalised. Time is rescaled with the typical time taken to fully (dis)charge the cathode at a current of size $\hat{I}$, namely

$$
\tau=\frac{L A \epsilon_{p} F \hat{c}_{c}}{\hat{I}},
$$

and the macroscopic spatial dimension, $x^{*}$, is scaled with the electrode width $L$. Electrolyte diffusivity, conductivity and concentration are scaled with their typical values, $\hat{\mathcal{B}}, \hat{D}, \hat{\kappa}$ and $\hat{c}$, respectively. Lithium concentration in the electrode particles is scaled with its maximum concentration $\hat{c}_{c}$. The various currents and current densities are all scaled based on the size of the current being supplied to/drawn from the external circuit. The scaling for the ionic flux is based on the typical size of its diffusive component which, for concentrations of size $\hat{c}$, length-scales of size $L$ and effective diffusivities of size $\hat{\mathcal{B}} \hat{D} / \epsilon_{l}$ is $\hat{\mathcal{B}} \hat{D} \hat{c} / \epsilon_{l} L^{2}$. The BV reaction rates are scaled based on the average flux required through particle surfaces needed to sustain a current of size $\hat{I}$. The typical size of the variations in the different potentials across the electrode are on the order of the thermal voltage. In the cases of the electrolyte and cathode potentials this is by design, and results from the relatively high conductivities of electrolytes and binders used in battery applications. The over-potential, $U_{c}^{*}$, is very close to being constant $\left(\hat{U}_{c} \approx 3.4 \mathrm{~V}\right)$ throughout most of the material's range of charge states, cf figure 2 . We therefore scale the over-potential by first subtracting off this constant contribution and allow for variations on the size of thermal voltage. In summary, the rescalings read

$$
\begin{array}{llll}
t^{*}=\tau t & x^{*}=L x & c^{*}=\hat{c} c & c_{c}^{*}=\hat{c}_{c} c_{c} \\
j^{*}=\frac{\hat{I}}{A} j & j_{c}^{*}=\frac{\hat{I}}{A} j_{c} & \phi^{*}=\frac{R_{g} T}{F} \phi & \phi_{c}^{*}=\hat{U}_{c}+\frac{R_{g} T}{F} \phi_{c} \\
\eta^{*}=\frac{R_{g} T}{F} \eta & U_{c}^{*}=\hat{U}_{c}+\frac{R_{g} T}{F} U_{c} & V^{*}=\frac{R_{g} T}{F} V & G^{*}=\frac{\hat{I}}{A L F b_{c}} G \\
I^{*}=\hat{I} I & \mathcal{F}^{*}=\frac{\hat{\mathcal{B}} \hat{D} \hat{c}}{L} \mathcal{F} & D^{*}=\hat{D} D & \mathcal{B}^{*}=\hat{\mathcal{B} \mathcal{B}} \\
\kappa^{*}=\hat{\kappa} \kappa & & &
\end{array}
$$

The non-dimensionalisation gives rise to the following dimensionless quantities that characterise the system:

$$
\begin{array}{llll}
\mathcal{N}=\frac{L^{2} \epsilon_{l}}{\tau \hat{\mathcal{B}} \hat{D}}, & \Gamma=\frac{\hat{I} L}{\hat{c} \hat{D} \hat{\mathcal{B}} A F}, & \Upsilon=\frac{k_{0} \hat{c}^{1 / 2} \hat{c}_{c} F A L b_{c}}{\hat{I}}, & \mathcal{L}=\frac{L_{s}}{L}, \\
\Theta=\frac{\sigma_{c} R_{g} T A}{F L \hat{I}}, & \mathcal{R}=\frac{R_{c} F \hat{I}}{R_{g} T}, & \mathcal{P}=\frac{\hat{\mathcal{B}} \hat{\kappa} R_{g} T A}{F L \hat{I}} .
\end{array}
$$

Here, $\mathcal{N}$ is the timescale for liquid diffusion over the timescale for cell discharge, $\Gamma$ is the ratio of the typical sizes of the drift flux to the diffusive flux, $\Upsilon$ is the typical current density at the surface of an electrode particle over the ratio of the exchange current density, $\mathcal{L}$ is the length of the separator over the length of the cathode, $\Theta$ is the electronic conductivity over the characteristic conductivity of the electrode particles, $\mathcal{R}$ is the voltage dropped at 
the current collector over the potential difference between fully charged and fully discharged electrode particles, and $\mathcal{P}$ is the ionic conductivity over the characteristic conductivity of the electrode particles.

\subsection{The Dimensionless Problem}

The system of equations obtained by applying the rescalings (16)-(20) to the variables (1)(13) is

$$
\begin{gathered}
\mathcal{N} \frac{\partial c}{\partial t}+\frac{\partial \mathcal{F}}{\partial x}=0, \quad \mathcal{F}=-\mathcal{B}(x) D(c) \frac{\partial c}{\partial x}-\Gamma\left(1-t^{+}\right) j \\
\frac{\partial j}{\partial x}=G, \quad j=-\mathcal{P} \mathcal{B}(x) \kappa(c)\left(\frac{\partial \phi}{\partial x}-2 \frac{1-t^{+}}{c} \frac{\partial c}{\partial x}\right), \\
\frac{\partial j_{c}}{\partial x}=-G, \quad j_{c}=-\Theta \frac{\partial \phi_{c}}{\partial x}, \quad \frac{d c_{c}}{d t}=-G, \\
G=\Upsilon c^{1 / 2} c_{c}^{1 / 2}\left(1-c_{c}\right)^{1 / 2}\left(\exp \left(\frac{\eta}{2}\right)-\exp \left(\frac{-\eta}{2}\right)\right), \quad \eta=\phi_{c}-\phi,
\end{gathered}
$$

subject to boundary conditions

$$
\begin{array}{r}
\left.j\right|_{x=-\mathcal{L}}=I(t),\left.\quad \mathcal{F}\right|_{x=-\mathcal{L}}=0,\left.\quad \phi\right|_{x=-\mathcal{L}}=0, \\
\left.\mathcal{F}\right|_{x=1}=0,\left.\quad j\right|_{x=1}=0,\left.\quad j_{c}\right|_{x=1}=I(t),
\end{array}
$$

and initial conditions

$$
\left.c\right|_{t=0}=1,\left.\quad c_{c}\right|_{t=0}=\alpha .
$$

The dimensionless cell voltage is given by

$$
V=\left.\phi_{c}\right|_{x=1}-\mathcal{R} I(t)
$$

For an LFP half-cell, $U_{c}$, the dimensionless variation in the over-potential is so small that it can be set to zero without engendering any significant errors, provided that we do not demand accuracy at states of charge where the over-potential varies rapidly and very significantly (i.e. states at which the particles approach full lithiation). In the equations above we have therefore set $U_{c}=0$ and it will not appear in the subsequent analysis.

Features of the model It will turn out to be helpful to note some generic features exhibited by the model equations. The total current density (made up of both an ionic and electronic component) is uniform throughout the electrode. On summing equations (24a) and (25a), integrating and applying boundary conditions (28b,c) we arrive at

$$
j+j_{c}=I(t) .
$$


Furthermore, integrating equation (25a) with respect to $x$ and again applying boundary conditions (27a) and (28c) leads to

$$
\int_{0}^{1} G d x=-I(t),
$$

indicating that the total reaction through the electrode is in balance with the current supplied by or driven from the external circuit.

\subsection{Dimensionless parameter estimates}

We will consider two device parameterisations; one for modern electrodes and one for older electrodes representative of the devices that were being produced in the early 2000s. As alluded to in the $\S 1$, a key feature in the development of LFP cathodes has been the increase in the conductivity of the solid matrix by the addition of dopants and conductive additives. We therefore parameterise our two devices in the same way except that, the solid conductivity is taken to be significantly larger in the modern parameter set. It is by contrasting these two cases that we are able to elucidate why this development has been so impactful in manufacturing better electrodes and it allows us to suggest some avenues for further improvements.

A list of parameter estimates obtained from the literature is supplied in Table 1. Note that $\hat{\mathcal{B}}, \hat{D}$ and $\hat{\kappa}$ are the typical values of the diffusivity and ionic conductivity respectively, cf (221)-(223) and figure 2. Specifically we choose $\hat{\mathcal{B}}=0.3150, \hat{D}=5.253 \times 10^{-10} \mathrm{~m}^{2} \mathrm{~s}^{-1}$, and

$\hat{\kappa}=1.088 \mathrm{~S} \mathrm{~m}^{-1}$. Based on this data the dimensionless parameters for the older electrodes being operated at a $\mathrm{C}$-rate of $1 \mathrm{C}$, the non-dimensional parameters are

$$
\begin{array}{llll}
\text { For 1C: } & \mathcal{N}=0.0028, & \Gamma=0.0602, & \Upsilon=113.8420, \\
\mathcal{L}=0.4167, & \Theta=0.1335, & \mathcal{R}=0.0223, & \mathcal{P}=9.1498 .
\end{array}
$$

Adopting the modern parameter set we find that a cell (dis)charging at a C-rate of 1, the parameter values are

$$
\begin{array}{llll}
\text { For 1C: } & \mathcal{N}=0.0028, & \Gamma=0.0602, & \Upsilon=113.8420, \\
\mathcal{L}=0.4167, & \Theta=93.1975, & \mathcal{R}=0.0223, & \mathcal{P}=9.1498 .
\end{array}
$$

For a more aggressive rate of $10 \mathrm{C}$ they are

$$
\begin{array}{llll}
\text { For 10C: } & \mathcal{N}=0.0280, & \Gamma=0.6022, & \Upsilon=11.3842, \\
\mathcal{L}=0.4167, & \Theta=9.3198, & \mathcal{R}=0.2234, & \mathcal{P}=0.9150 .
\end{array}
$$

Finally, for a very aggressive rate of $100 \mathrm{C}$ they are

$$
\begin{array}{llll}
\text { For 100C: } & \mathcal{N}=0.2798, & \Gamma=6.0222, & \Upsilon=1.1384, \\
\mathcal{L}=0.4167, & \Theta=0.9320, & \mathcal{R}=2.2344, & \mathcal{P}=0.0915
\end{array}
$$

We note that the only difference between the modern device and the older device at $1 \mathrm{C}$ is the value of $\Theta$, the dimensionless solid conductivity, of (34) and (36). The value of the current demand at $1 \mathrm{C}$, e.g. , can be found by solving (15) for $\hat{I}$ having specified $\tau=3600 \mathrm{~s}$, which gives $\hat{I}=0.1603 \mathrm{~A}$. Current demands for other C-rates can be found similarly. 


\section{Numerical solution of the DFN model}

In order to motivate and verify the asymptotic analysis of $\S 4$ we present some representative numerical solutions of cathode discharge. A variety of numerical methods can be employed to obtain approximate solutions to the model but here we use the method of lines and the MATLAB integrator ode15s to obtain numerical solutions. The approach is chosen because it is relatively simple to implement, cheap to use in terms of computing requirements, and gives us the requisite accuracy. First, the derivatives in the macroscopic spatial dimension, $x$, are approximated using finite element method, this results in a large system of differential algebraic equations (DAEs). The differential parts of this system arise from the parabolic PDEs whereas the algebraic equations arise from the elliptic PDEs. The system of DAEs can then be integrated forward in time using the ode15s integrator in MATLAB. The method is loosely based on the approach described in [43] and is currently under development as a piece of software code named DandeLiion [23]. We refer the interested reader to those publications for an in-depth description of the method.

In figures 3-6 we present four representative solutions, one for each of the parameter sets given in $\S 2.3$, and subjecting the cathode to galvanostatic discharge from a state of charge with $\alpha=0.035$ until discharge terminates either because the voltage drops below $2.5 \mathrm{~V}$, or the electrolyte becomes depleted. In all four cases we observe that the reaction is initially concentrated around the edges of the electrode (where the particles lithiate rapidly). Once the particles at these extremities are close to being fully lithiated the reaction progresses into the bulk of the electrode. We see that, for the older electrode in figure 3 , the region in which the reaction occurs remains narrow and it propagates from the current collect towards the separator. By contrast, for the modern electrode, cf figures 4-6, the reaction fronts are considerably more diffuse, though the width of the layers does decrease with increasing C-rate. At the highest discharge rate the large current leads to electrolyte depletion near the current collector and terminates discharge before the cell can be fully utilised. We note also that in the modern electrodes the reaction front propagates in the opposite direction, beginning at the separator and proceeding towards the current collector. The presence of the narrow reaction front, which is most clearly visible for the parameterisation relevant to older electrodes, is what motivates the asymptotic analysis in the subsequent section.

\section{Asymptotic analysis in the limit $\Upsilon \rightarrow \infty$}

The computations shown in the previous section suggest that an asymptotic regime pertains to the set of parameter values relevant for older electrode designs. We will now show that this regime is in fact a distinguished limit characterised by the large value of $\Upsilon$. For large values of $\Upsilon$ the generic solution structure has a wide region of slowly reacting electrode particles in the bulk separated from regions of fully lithiated particles by narrow rapidly reacting regions which intrude from the edges of the electrode into its interior as discharge proceeds. We note that only one of the reaction fronts (that propagating inwards from the current collector) is clearly visible in the computation shown in figure 3. As will shortly become clear, this is because older electrode designs also feature an extreme contrast in the conductivity of electrolyte compared to the solid matrix (giving rise to $\mathcal{P}>\Theta$ ) and this 

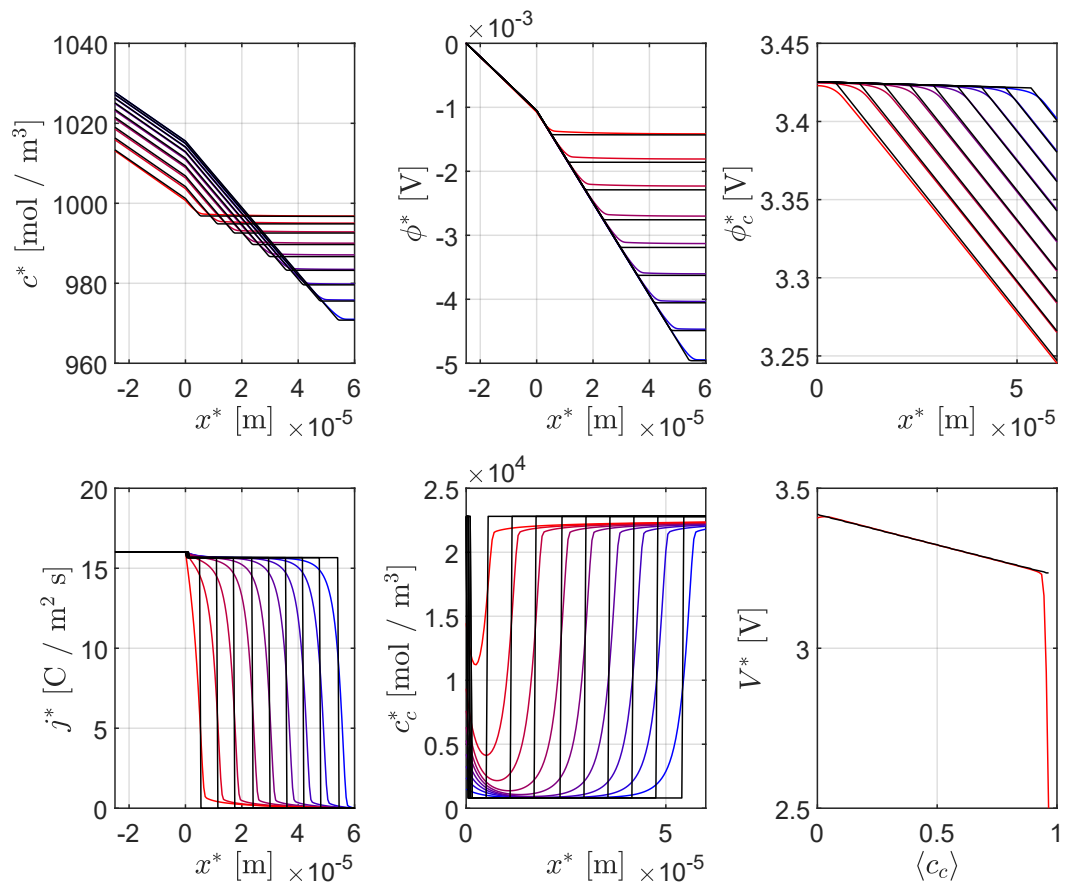

Figure 3: Discharge of an older electrode at 1C. Figure showing the comparison between numerical simulations of the full DFN model (coloured) and the solution predicted by the asymptotic RFM (black). Plots for $c^{*}, j^{*}, \phi^{*}, \phi_{c}^{*}$, and $c_{c}^{*}$ are taken at times $t \in\{0.1,0.2,0.3,0.4,0.5,0.6,0.7,0.8,0.9\}$, where plots fade from blue to red as time increases, as well as the voltage profile $V^{*}$. The parameter values are those shown in (34). Since we are considering galvanostatic discharge, the value of $\left\langle c_{c}\right\rangle$ on the horizontal axis of the bottom-right figure is interchangeable with $t$. 

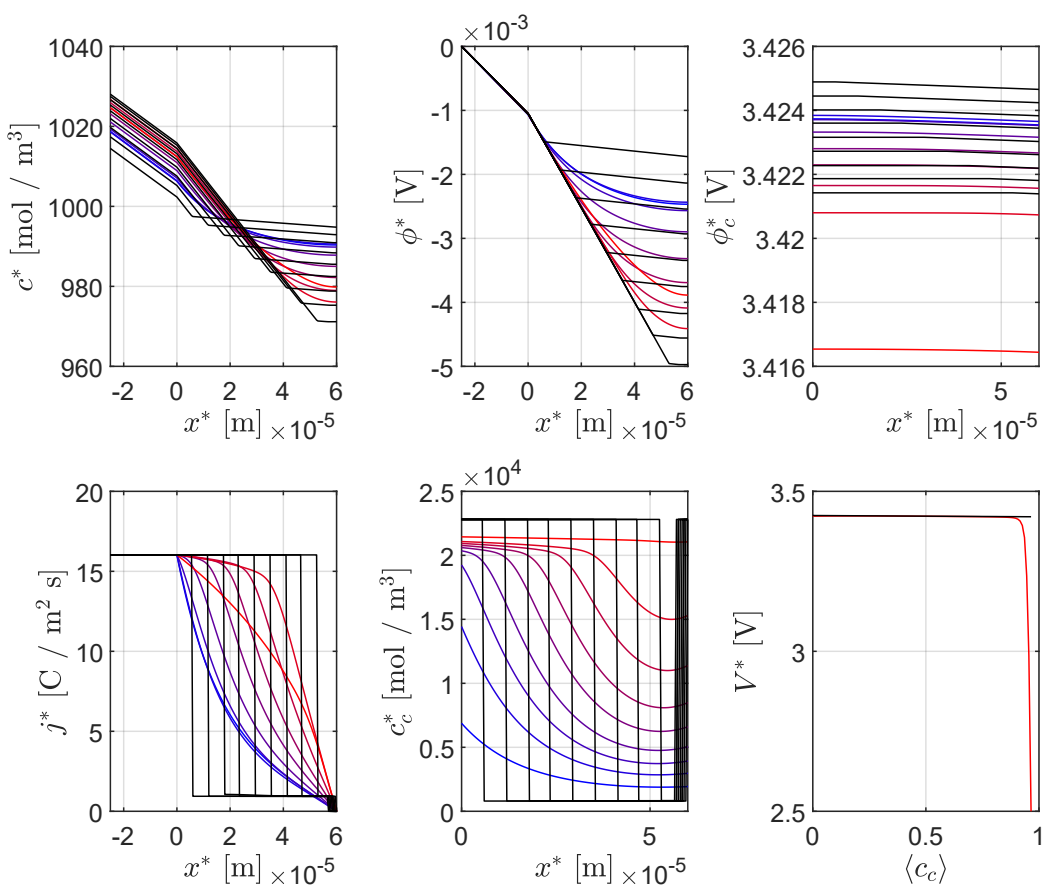

Figure 4: Figure showing the comparison between numerical simulations of the full DFN model (coloured) and the solution predicted by the asymptotic RFM (black). Plots for $c^{*}$, $j^{*}, \phi^{*}, \phi_{c}^{*}$, and $c_{c}^{*}$ are taken at times $t \in\{0.1,0.2,0.3,0.4,0.5,0.6,0.7,0.8,0.9\}$, where plots fade from blue to red as time increases, as well as the voltage profile $V^{*}$. The parameters values are those shown in (36) and table 1 for a C-rate of $1 \mathrm{C}$ using the modern electrode value for the matrix conductivity. 

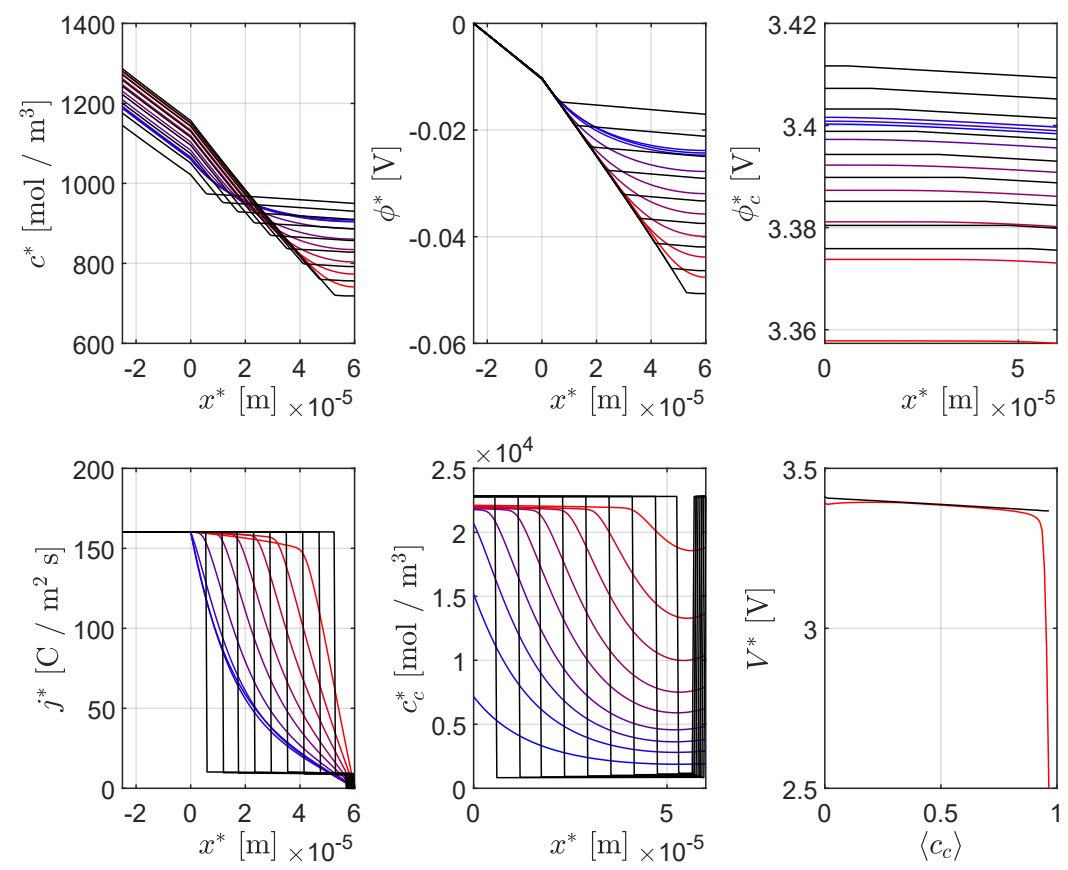

Figure 5: Figure showing the comparison between numerical simulations of the full DFN model (coloured) and the solution predicted by the asymptotic RFM (black). Plots for $c^{*}$, $j^{*}, \phi^{*}, \phi_{c}^{*}$, and $c_{c}^{*}$ are taken at times $t \in\{0.1,0.2,0.3,0.4,0.5,0.6,0.7,0.8,0.9\}$, where plots fade from blue to red as time increases, as well as the voltage profile $V^{*}$. The parameters values are those shown in (38) and table 1 for a C-rate of 10C using the modern electrode value for the matrix conductivity. 

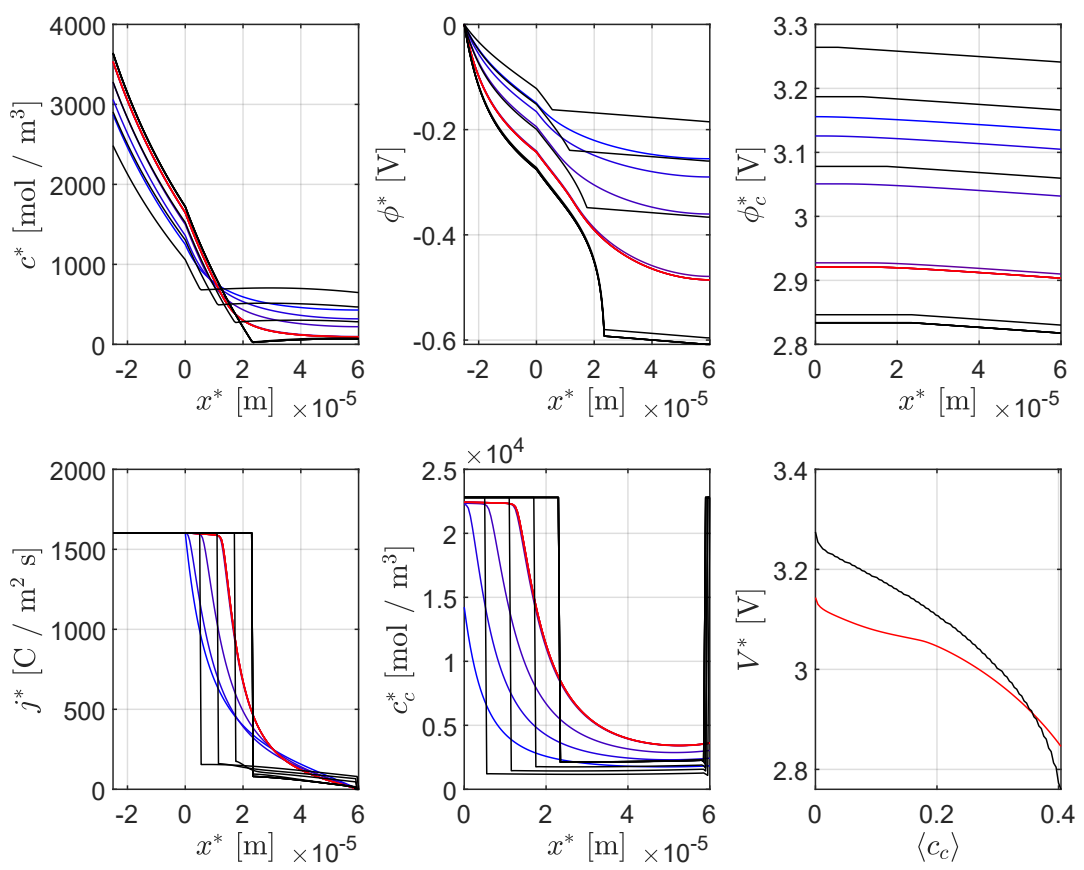

Figure 6: Figure showing the comparison between numerical simulations of the full DFN model (coloured) and the solution predicted by the asymptotic RFM (black). Plots for $c^{*}$, $j^{*}, \phi^{*}, \phi_{c}^{*}$, and $c_{c}^{*}$ are taken at times $t \in\{0.1,0.2,0.3,0.4\}$, where plots fade from blue to red as time increases, as well as the voltage profile $V^{*}$. The parameters values are those shown in (40) and table 1 for a C-rate of $100 \mathrm{C}$ using the modern electrode value for the matrix conductivity. 

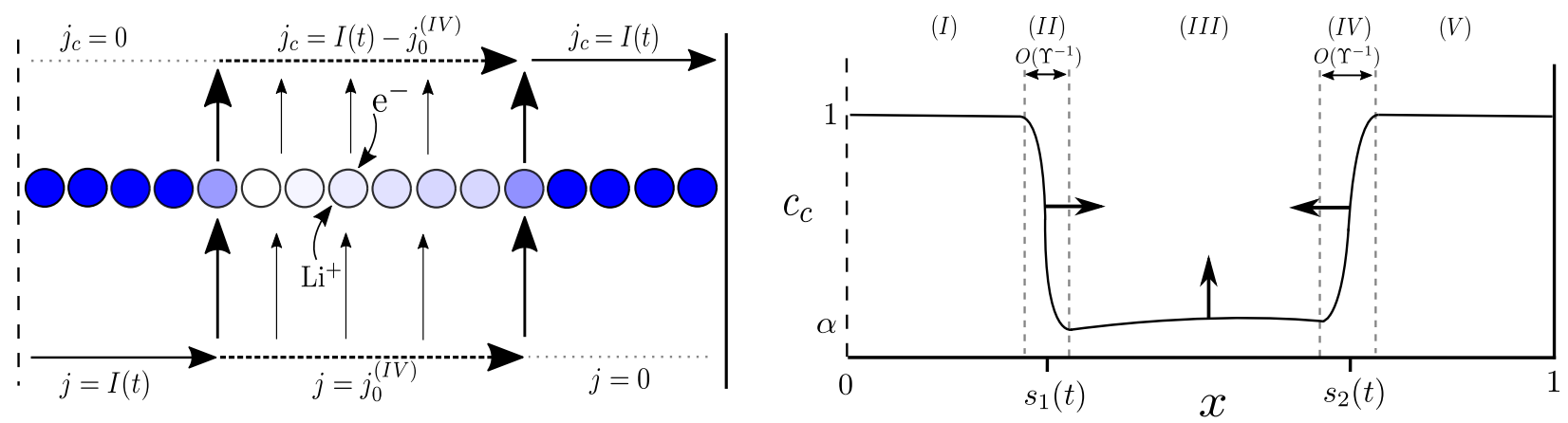

Figure 7: Left: Sketch of the electrode showing the structure of the solution and the locations of the predominant reactions. Right: The same electrode annotated with the notation for the different asymptotic regions and locations of the reaction fronts.

suppresses the motion of the reaction that would otherwise propagate from the separator. In the modern electrodes the opposite is true, i.e. $\Theta>\mathcal{P}$, and this leads to suppression of the reaction front moving from the current collector towards the separator. This generic behaviour with two moving reaction fronts can be seen in the computations that we will use to verify the asymptotic analysis, cf figures 9-10.

Formally, we shall consider the limit in which $\Upsilon \rightarrow \infty$ and all other parameters will be taken to be $O(1)$. The solution is described by five asymptotic regions which we will distinguish using Roman numeral superscripts as follows: the central slowly reacting region (denoted region $(I I I)$ ), the two reacted regions near the separator and current collector (termed regions $(I)$ and region $(V)$ respectively), and the narrow moving reaction fronts positioned at $x=s_{1}(t)$ and $x=s_{2}(t)$ (denoted regions $(I I)$ and $(I V)$ ) respectively. A sketch of this structure in shown in Fig. 7. Readers interested in the result of the asymptotic analysis are pointed to $\S 5$ where the upshot of the analysis, namely the reduced order model (or reaction front model, $\mathrm{RFM}$ ), is summarised.

\subsection{Outer regions}

The outer regions, namely region $(I)$ where $-\mathcal{L}<x<s_{1}(t)$, region $(I I I)$ where $s_{1}(t)<x<$ $s_{2}(t)$, and region $(V)$ where $s_{2}(t)<x<1$, are analysed on the length-scale of the electrode.

Region $(I)$ : reacted region adjacent to separator In region $(I)$, where $-\mathcal{L}<x<$ $s_{1}(t)$, the particles are almost fully lithiated and consequently it is unable to sustain a significant reaction or convert a significant portion of the ionic current (which enters the electrode via the adjacent separator) into electronic form. It follows, from (31), that the electronic current in this region is small and thus the potential gradient in the solid must also be significantly smaller than that across the electrolyte in this region. The asymptotic 
solution in this region is found by expanding as follows:

$$
\begin{aligned}
c^{(I)} & =c_{0}^{(I)}(x, t)+\cdots & & \mathcal{F}^{(I)}=\mathcal{F}_{0}^{(I)}(x, t)+\cdots \\
j^{(I)} & =I(t)+\cdots & & j_{c}^{(I)}=o(1) \\
\phi^{(I)} & =\phi_{0}^{(I)}(x, t)+\cdots & & \phi_{c}^{(I)}=\phi_{c, 0}^{(I)}(t)+\cdots \\
c_{c}^{(I)} & =1+\cdots & & G^{(I)}=o(1) .
\end{aligned}
$$

Here we use the superscript $(I)$ to indicate variables within this region. Equations (24a), (25), and (26) are automatically satisfied at leading order by the expansions (41)-(44). The three remaining leading order variables $c_{0}^{(I)}, \mathcal{F}_{0}^{(I)}$ and $\phi_{0}^{(I)}$ satisfy equations that are obtained by balancing the leading order terms in (23) and (24b); these read:

$$
\begin{array}{r}
\mathcal{N} \frac{\partial c_{0}^{(I)}}{\partial t}+\frac{\partial \mathcal{F}_{0}^{(I)}}{\partial x}=0, \quad \mathcal{F}_{0}^{(I)}=-\mathcal{B}(x) D\left(c_{0}^{(I)}\right) \frac{\partial c_{0}^{(I)}}{\partial x}-\Gamma\left(1-t^{+}\right) I(t) \\
I(t)=-\mathcal{P} \mathcal{B}(x) \kappa\left(c_{0}^{(I)}\right)\left(\frac{\partial \phi_{0}^{(I)}}{\partial x}-2 \frac{1-t^{+}}{c_{0}^{(I)}} \frac{\partial c_{0}^{(I)}}{\partial x}\right) .
\end{array}
$$

and satisfy the boundary conditions

$$
\left.\mathcal{F}_{0}^{(I)}\right|_{x=-\mathcal{L}}=0,\left.\quad \phi_{0}^{(I)}\right|_{x=-\mathcal{L}}=0,
$$

on the separator (obtained from $(27 b, c)$ and at leading order).

Region $(V)$ : reacted region adjacent to current collector In the other reacted region, lying in $s_{2}(t)<x<1$, the asymptotic expansion proceeds analogously as it does in region $(I)$ (for similar reasons) with the notable exception that the current flows almost entirely in the solid electrode matrix (rather than the electrolyte); it reads:

$$
\begin{aligned}
& c^{(V)}=c_{0}^{(V)}(x, t)+\cdots \\
& \mathcal{F}^{(V)}=\mathcal{F}_{0}^{(V)}(x, t)+\cdots \\
& j^{(V)}=o(1) \\
& j_{c}^{(V)}=I(t)+\cdots \\
& \phi^{(V)}=\phi_{0}^{(V)}(x, t)+\cdots \\
& \phi_{c}^{(V)}=\phi_{c, 0}^{(V)}(x, t)+\cdots \\
& c_{c}^{(V)}=1+\cdots \\
& G^{(V)}=o(1)
\end{aligned}
$$

Equations (24a), (25a), (25c)-(26) are automatically satisfied at leading order by the expansions (48)-(51). The remaining leading order variables, $c_{0}^{(V)}, \mathcal{F}_{0}^{(V)}, \phi_{0}^{(V)}$, and $\phi_{c, 0}^{(V)}$ satisfy the leading balances in equations (23), (24b), (25b) which read;

$$
\begin{array}{r}
\mathcal{N} \frac{\partial c_{0}^{(V)}}{\partial t}+\frac{\partial \mathcal{F}_{0}^{(V)}}{\partial x}=0, \quad \mathcal{F}_{0}^{(V)}=-\mathcal{B}(x) D\left(c_{0}^{(V)}\right) \frac{\partial c_{0}^{(V)}}{\partial x} \\
0=-\mathcal{P B}(x) \kappa\left(c_{0}^{(V)}\right)\left(\frac{\partial \phi_{0}^{(V)}}{\partial x}-2 \frac{1-t^{+}}{c_{0}^{(V)}} \frac{\partial c_{0}^{(V)}}{\partial x}\right), \quad-I(t)=\Theta \frac{\partial \phi_{c, 0}^{(V)}}{\partial x}
\end{array}
$$

along with the boundary condition

$$
\left.\mathcal{F}_{0}^{(V)}\right|_{x=1}=0,
$$

on the current collector (obtained from (28a) at leading order). 
Region $(I I I)$ : central slowly reacting region In the central slowly reacting region, lying in $s_{1}(t)<x<s_{2}(t)$, there are significant current flows in both the electrolyte and the solid and significant charge transfer is occurring, i.e. there is an $O(1)$ amount of reaction in this region. Since the electrode particles here still have space to accommodate more $\mathrm{Li}^{+}$, the only way in which $(26)$ is consistent with $O(1)$ reaction rates is if the potentials in the electrolyte and solid are almost equal. The asymptotic solution can be found by expanding as follows

$$
\begin{aligned}
c^{(I I I)} & =c_{0}^{(I I I)}(x, t)+\cdots & & \mathcal{F}^{(I I I)}=\mathcal{F}_{0}^{(I I I)}(x, t)+\cdots \\
j^{(I I I)} & =j_{0}^{(I I I)}(x, t)+\cdots & & j_{c}^{(I I I)}=I(t)-j_{0}^{(I I I)}(x, t)+\cdots \\
\phi^{(I I I)} & =\phi_{0}^{(I I I)}(x, t)+\cdots & & \phi_{c}^{(I I I)}=\phi_{0}^{(I I I)}(x, t)+\cdots \\
c_{c}^{(I I I)} & =c_{c, 0}^{(I I I)}(x, t)+\cdots & & G^{(I I I)}=G_{0}^{(I I I)}(x)+\cdots .
\end{aligned}
$$

On substituting this expansion into (23)-(26) we find that the leading order variables $c_{0}^{(I I I)}$, $\mathcal{F}_{0}^{(I I I)}, j_{0}^{(I I I)}, \phi_{0}^{(I I I)}, c_{c, 0}^{(I I I)}$, and $G_{0}^{(I I I)}$ satisfy the following equations

$$
\begin{array}{r}
\mathcal{N} \frac{\partial c_{0}^{(I I I)}}{\partial t}+\frac{\partial \mathcal{F}_{0}^{(I I I)}}{\partial x}=0, \quad \mathcal{F}_{0}^{(I I I)}=-\mathcal{B}(x) D\left(c_{0}^{(I I I)}\right) \frac{\partial c_{0}^{(I I I)}}{\partial x}-\Gamma\left(1-t^{+}\right) j_{0}^{(I I I)} \\
j_{0}^{(I I I)}=-\mathcal{P} \mathcal{B}(x) \kappa\left(c_{0}^{(I I I)}\right)\left(\frac{\partial \phi_{0}^{(I I I)}}{\partial x}-2 \frac{1-t^{+}}{c_{0}^{(I I I)}} \frac{\partial c_{0}^{(I I I)}}{\partial x}\right), \quad \Theta \frac{\partial \phi_{0}^{(I I I)}}{\partial x}=j_{0}^{(I I I)}-I(t), \\
\frac{\partial c_{c, 0}^{(I I I)}}{\partial t}=-G_{0}^{(I I I)}, \quad \frac{\partial j_{0}^{(I I I)}}{\partial x}=G_{0}^{(I I I)},
\end{array}
$$

By eliminating the leading order potential gradient between equations (60a) and (60b) we can solve for the ionic current in terms of the leading order electrolyte concentration, yielding

$$
j_{0}^{(I I I)}=\frac{\mathcal{P} \mathcal{B}(x) \kappa\left(c_{0}^{(I I I)}\right)}{\Theta+\mathcal{P B}(x) \kappa\left(c_{0}^{(I I I)}\right)}\left(I(t)+2 \Theta \frac{1-t^{+}}{c_{0}^{(I I I)}} \frac{\partial c_{0}^{(I I I)}}{\partial x}\right) .
$$

We can then solve for the lithium content in the solid in terms of the ionic current from by eliminating $G_{0}^{(I I I)}$ equation (61), integrating with respect to time $t$ and applying the initial condition (29b), giving:

$$
c_{c, 0}^{(I I I)}=\alpha-\int_{0}^{t} \frac{\partial j_{0}^{(I I I)}}{\partial x} d t^{\prime}
$$

\subsection{The narrow reaction regions}

We now investigate the narrow reaction regions, namely regions $(I I)$ where $x-s_{1}(t)=$ $O\left(\Upsilon^{-1}\right)$ and $(I V)$ where $x-s_{2}(t)=O\left(\Upsilon^{-1}\right)$, separating the three outer regions discussed above. 
Region $(I I)$ : left-hand reaction region The left-hand reaction region, located about $x=s_{1}(t)$, has width $O\left(\Upsilon^{-1}\right)$, and is therefore studied under a change of coordinates given by

$$
x-s_{1}(t)=\Upsilon^{-1} y,
$$

and so the local equations become

$$
\begin{gathered}
\mathcal{N}\left(-\Upsilon \dot{s}_{1}(t) \frac{\partial c^{(I I)}}{\partial y}+\frac{\partial c^{(I I)}}{\partial t}\right)+\Upsilon \frac{\partial \mathcal{F}^{(I I)}}{\partial y}=0, \quad \mathcal{F}^{(I I)}=-\Upsilon \mathcal{B}(x) D\left(c^{(I I)}\right) \frac{\partial c^{(I I)}}{\partial y}-\Gamma\left(1-t^{+}\right) j^{(I I)} \\
\Upsilon \frac{\partial j^{(I I)}}{\partial y}=G^{(I I)}, \quad j^{(I I)}=-\Upsilon \mathcal{P} \mathcal{B}(x) \kappa\left(c^{(I I)}\right)\left(\frac{\partial \phi^{(I I)}}{\partial y}-2 \frac{1-t^{+}}{c^{(I I)}} \frac{\partial c^{(I I)}}{\partial y}\right) \\
\Upsilon \frac{\partial j_{c}^{(I I)}}{\partial y}=-G^{(I I)}, \quad j_{c}^{(I I)}=-\Upsilon \Theta \frac{\partial \phi_{c}^{(I I)}}{\partial y}, \quad-\Upsilon \dot{s}_{1}(t) \frac{\partial c_{c}^{(I I)}}{\partial y}+\frac{\partial c_{c}^{(I I)}}{\partial t}=-G^{(I I)} \\
G^{(I I)}=\Upsilon \sqrt{c^{(I I)} c_{c}^{(I I)}\left(1-c_{c}^{(I I)}\right)}\left(e^{\frac{\eta}{2}}-e^{-\frac{\eta}{2}}\right), \quad \eta=\phi_{c}^{(I I)}-\phi^{(I I)}
\end{gathered}
$$

The asymptotic solution can then be found by expanding as follows

$$
\begin{array}{lll}
c^{(I I)}=c_{0}^{(I I)}(t)+\cdots, & \mathcal{F}^{(I I)}=\mathcal{F}_{0}^{(I I)}(t)+\cdots, \\
j^{(I I)}=j_{0}^{(I I)}(y, t)+\cdots, & \phi^{(I I)}=\phi_{0}^{(I I)}(t)+\cdots, \\
j_{c}^{(I I)}=I(t)-j_{0}^{(I I)}(y, t)+\cdots, & \phi_{c}^{(I I)}=\phi_{c, 0}^{(I I)}(t)+\cdots \\
c_{c}^{(I I)}=c_{c, 0}^{(I I)}(y, t)+\cdots, & & G^{(I I)}=\Upsilon G_{-1}^{(I)}(y, t)+\cdots,
\end{array}
$$

which, by design, satisfy equations (65)-(66) and (67b). On substituting these expansions into (67c)-(68) we find that the leading order variables $j_{0}^{(I I)}$ and $c_{c, 0}^{(I I)}$ satisfy the following equations:

$$
-\dot{s}_{1}(t) \frac{\partial c_{c, 0}^{(I I)}}{\partial y}=2 \sqrt{c_{0}^{(I I)}(t) c_{c, 0}^{(I I)}\left(1-c_{c, 0}^{(I I)}\right)} \sinh \left(\frac{\phi_{c, 0}^{(I I)}-\phi_{0}^{(I I)}}{2}\right), \quad \dot{s}_{1}(t) \frac{\partial c_{c, 0}^{(I I)}}{\partial y}=-\frac{\partial j_{0}^{(I I)}}{\partial y} .(70)
$$

Matching across the left-hand reaction region Upon matching the leading order lithium concentrations and fluxes (in the electrolyte) across the regions adjacent to the lefthand reaction front situated about $x=s_{1}(t)$ we find that

$$
\begin{gathered}
\left.c_{0}^{(I)}\right|_{x>_{s_{1}(t)}}=c_{0}^{(I I)}(t)=\left.c_{0}^{(I I I)}\right|_{x \searrow s_{1}(t)}, \\
\left.\mathcal{F}_{0}^{(I)}\right|_{x \gamma_{s_{1}(t)}=\mathcal{F}_{0}^{(I I)}(t)}=\left.\mathcal{F}_{0}^{(I I I)}\right|_{x \searrow s_{1}(t)} .
\end{gathered}
$$

The equations for the concentration and flux of lithium in the electrolyte however cannot be closed at this stage; this will be done in $\S 4.3$ after matching across the right-hand reaction front around $x=s_{2}(t)$. Performing a similar operation for the leading order electrolyte potentials we obtain the following matching conditions:

$$
\left.\phi_{0}^{(I)}\right|_{x \nearrow_{s_{1}(t)}}=\phi_{0}^{(I I)}(t)=\left.\phi_{0}^{(I I I)}\right|_{x \searrow s_{1}(t)} .
$$


On matching the leading order electronic potentials we find

$$
\phi_{c, 0}^{(I)}(t)=\phi_{c, 0}^{(I I)}(t)=\left.\phi_{c, 0}^{(I I I)}\right|_{x \searrow s_{1}(t)} .
$$

Matching the leading order lithium content inside the electrode particles across all three regions leads to the conditions

$$
\left.c_{c, 0}^{(I I)}\right|_{y \rightarrow-\infty}=1,\left.\quad c_{c, 0}^{(I I)}\right|_{y \rightarrow \infty}=\left.c_{c, 0}^{(I I I)}\right|_{x \searrow s_{1}(t)},
$$

Finally, the matching conditions on the leading order ionic current densities $j$ are

$$
\left.j_{0}^{(I I)}\right|_{y \rightarrow-\infty}=I(t),\left.\quad j_{0}^{(I I)}\right|_{y \rightarrow \infty}=\left.j_{0}^{(I I I)}\right|_{x \searrow_{s_{1}}(t) .} .
$$

An ODE which can be solved to determine the position of the reaction layer, $x=s_{1}(t)$, is found by integrating (70b) and imposing the conditions (75)-(76), giving

$$
\dot{s}_{1}(t)=\frac{\left.j_{0}^{(I I)}\right|_{y \rightarrow \infty}-\left.j_{0}^{(I I)}\right|_{y \rightarrow-\infty}}{\left.c_{c, 0}^{(I I)}\right|_{y \rightarrow \infty}-\left.c_{c, 0}^{(I I)}\right|_{y \rightarrow-\infty}}=\frac{I(t)-\left.j_{0}^{(I I I)}\right|_{x \searrow_{s_{1}}(t)}}{1-\left.c_{c, 0}^{(I I I)}\right|_{x \searrow_{s_{1}(t)}}}
$$

with the initial condition $\left.s_{1}(t)\right|_{t=0}=0$, which corresponds to the fact that this reaction region begins at the separator.

Region $(I V)$ : right-hand reaction region The right-hand reaction layer located about $x=s_{2}(t)$ also has width $O\left(\Upsilon^{-1}\right)$, and is therefore studied under a change of coordinates given by

$$
x-s_{2}(t)=\Upsilon^{-1} z,
$$

and so the local equations become

$$
\begin{gathered}
\mathcal{N}\left(-\Upsilon \dot{s}_{2}(t) \frac{\partial c^{(I V)}}{\partial z}+\frac{\partial c^{(I V)}}{\partial t}\right)+\Upsilon \frac{\partial \mathcal{F}^{(I V)}}{\partial z}=0, \quad \mathcal{F}^{(I V)}=-\Upsilon \mathcal{B}(x) D\left(c^{(I V)}\right) \frac{\partial c^{(I V)}}{\partial z}-\Gamma\left(1-t^{+}\right) j^{(I V)}, \\
\Upsilon \frac{\partial j^{(I V)}}{\partial z}=G, \quad j^{(I V)}=-\Upsilon \mathcal{P B}(x) \kappa\left(c^{(I V)}\right)\left(\frac{\partial \phi^{(I V)}}{\partial z}-2 \frac{1-t^{+}}{c^{(I V)}} \frac{\partial c^{(I V)}}{\partial z}\right), \\
\Upsilon \frac{\partial j_{c}^{(I V)}}{\partial z}=-G^{(I V)}, \quad j_{c}^{(I V)}=-\Upsilon \Theta \frac{\partial \phi_{c}^{(I V)}}{\partial z}, \quad-\Upsilon \dot{s}_{2}(t) \frac{\partial c_{c}^{(I V)}}{\partial z}+\frac{\partial c_{c}^{(I V)}}{\partial t}=-G^{(I V)}, \\
G^{(I V)}=\Upsilon \sqrt{c^{(I V)} c_{c}^{(I V)}\left(1-c_{c}^{(I V)}\right)}\left(e^{\frac{\eta}{2}}-e^{-\frac{\eta}{2}}\right), \quad \eta=\phi_{c}^{(I V)}-\phi^{(I V)}
\end{gathered}
$$

The asymptotic solution can then be found by expanding as follows

$$
\begin{array}{llrl}
c^{(I V)} & =c_{0}^{(I V)}(t)+\cdots, & \mathcal{F}^{(I V)} & =\mathcal{F}_{0}^{(I V)}(t)+\cdots, \\
j^{(I V)} & =j_{0}^{(I V)}(z, t)+\cdots, & \phi^{(I V)} & =\phi_{0}^{(I V)}(t)+\cdots, \\
j_{c}^{(I V)} & =I(t)-j_{0}^{(I V)}(z, t)+\cdots, & \phi_{c}^{(I V)} & =\phi_{c, 0}^{(I V)}(t)+\cdots, \\
c_{c}^{(V I)} & =c_{c, 0}^{(I V)}(z, t)+\cdots, & G^{(V I)} & =\Upsilon G_{-1}^{(I V)}(z, t)+\cdots,
\end{array}
$$


which, by design, satisfy equations (79)-(80) and (81b). On substituting these expansions into (81c)-(82) we find that the leading order variables $j_{0}^{(I V)}$ and $c_{c, 0}^{(I V)}$ satisfy the following equations:

$$
-\dot{s}_{2}(t) \frac{\partial c_{c, 0}^{(I V)}}{\partial z}=2 \sqrt{c_{0}^{(I V)}(t) c_{c, 0}^{(I V)}\left(1-c_{c, 0}^{(I V)}\right)} \sinh \left(\frac{\phi_{c, 0}^{(I V)}-\phi_{0}^{(I V)}}{2}\right), \quad \dot{s}_{2}(t) \frac{\partial c_{c, 0}^{(I V)}}{\partial z}=-\frac{\partial j_{0}^{(I V)}}{\partial z} .(84)
$$

Matching across the right-hand reaction region Upon matching the leading order lithium concentrations and fluxes (in the electrolyte) across the regions adjacent to the right-hand reaction front situated about $x=s_{2}(t)$ we find that

$$
\begin{gathered}
\left.c_{0}^{(I I I)}\right|_{x \nearrow s_{2}(t)}=c_{0}^{(I V)}(t)=\left.c_{0}^{(V)}\right|_{x \searrow s_{2}(t)}, \\
\left.\mathcal{F}_{0}^{(I I I)}\right|_{x \nearrow s_{2}(t)}=\mathcal{F}_{0}^{(I V)}(t)=\left.\mathcal{F}_{0}^{(V)}\right|_{x \searrow s_{2}(t)} .
\end{gathered}
$$

Performing a similar operation for the leading order electric potentials we obtain the following matching conditions:

$$
\left.\phi_{c, 0}^{(I I I)}\right|_{x \nearrow s_{2}(t)}=\phi_{c, 0}^{(I V)}(t)=\left.\phi_{c, 0}^{(V)}\right|_{x \searrow s_{2}(t)} .
$$

On matching the leading order electrolyte potentials we find

$$
\left.\phi_{0}^{(I I I)}\right|_{x \nearrow_{s_{2}(t)}}=\phi_{0}^{(I V)}(t)=\left.\phi_{0}^{(V)}\right|_{x \searrow s_{2}(t)} .
$$

Matching the leading order lithium content inside the electrode particles across all three regions leads to the conditions

$$
\left.c_{c, 0}^{(I I I)}\right|_{x \nearrow s_{2}(t)}=\left.c_{c, 0}^{(I V)}\right|_{z \rightarrow-\infty},\left.\quad c_{c, 0}^{(I V)}\right|_{z \rightarrow \infty}=1 .
$$

Finally, the matching conditions on the leading order ionic current densities $j$ are

$$
\left.j_{0}^{(I I I)}\right|_{x \nearrow s_{2}(t)}=\left.j_{0}^{(I V)}\right|_{z \rightarrow-\infty},\left.\quad j_{0}^{(I V)}\right|_{z \rightarrow \infty}=0 .
$$

The position of the first reaction layer $s_{2}(t)$ is found by integrating equation (84b) and applying conditions (89)-(90), giving

$$
\dot{s}_{2}(t)=\frac{\left.j_{0}^{(V I)}\right|_{w \rightarrow \infty}-\left.j_{0}^{(V I)}\right|_{w \rightarrow-\infty}}{\left.c_{c, 0}^{(V I)}\right|_{w \rightarrow \infty}-\left.c_{c, 0}^{(V I)}\right|_{w \rightarrow-\infty}}=-\frac{\left.j_{0}^{(I I I)}\right|_{x \nearrow s_{2}(t)}}{1-\left.c_{c, 0}^{(I I I)}\right|_{x \nearrow s_{2}(t)}},
$$

with the initial condition $\left.s_{2}(t)\right|_{t=0}=1$, which corresponds to the fact that this reaction region begins at the current collector.

\subsection{Matching between the outer regions}

A closed problem involving only the outer regions can now be formed. The matching conditions (71)-(72), (85)-(86) are

$$
\begin{gathered}
\left.c_{0}^{(I)}\right|_{x \nearrow s_{1}(t)}=\left.c_{0}^{(I I I)}\right|_{x \searrow s_{1}(t)},\left.\quad c_{0}^{(I I I)}\right|_{x \nearrow s_{2}(t)}=\left.c_{0}^{(V)}\right|_{x \searrow s_{2}(t)}, \\
\left.\mathcal{F}_{0}^{(I)}\right|_{x \nearrow s_{1}(t)}=\left.\mathcal{F}_{0}^{(I I I)}\right|_{x \searrow s_{1}(t)},\left.\quad \mathcal{F}_{0}^{(I I I)}\right|_{x \nearrow s_{2}(t)}=\left.\mathcal{F}_{0}^{(V)}\right|_{x \searrow s_{2}(t)} .
\end{gathered}
$$


which translate to imposing continuity of concentration and flux of the anions in the electrolyte across the narrow reaction fronts. An expression for the ionic current densities in terms of the ionic concentration in the outer regions is obtained by collating equations (42a), (49a), and (62), which read

$$
j_{0}^{(I)}=I(t), \quad j_{0}^{(I I I)}=\frac{\mathcal{P} \mathcal{B}(x) \kappa\left(c_{0}^{(I I I)}\right)}{\Theta+\mathcal{P B}(x) \kappa\left(c_{0}^{(I I I)}\right)}\left(I(t)+2 \Theta \frac{1-t^{+}}{c_{0}^{(I I I)}} \frac{\partial c_{0}^{(I I I)}}{\partial x}\right), \quad j_{0}^{(V)}=0 .
$$

\section{The reaction front model}

The main results of the asymptotic analysis can be summarised in the form of a simplified reduced order model, termed the reaction front model (RFM), that provides a good approximation of the DFN model in the distinguished limit $\Upsilon \rightarrow \infty$. This is presented below. The leading order ion transport equations in the outer regions (45), (52), and (59) read

$$
\mathcal{N} \frac{\partial c}{\partial t}+\frac{\partial \mathcal{F}}{\partial x}=0, \quad \mathcal{F}=-\mathcal{B}(x) D(c) \frac{\partial c}{\partial x}-\Gamma\left(1-t^{+}\right) j, \quad \text { in } \quad-\mathcal{L}<x<1,
$$

and since $c$ and $\mathcal{F}$ are continuous across the boundary layer regions separating regions $(I)$, $(I I I)$ and $(V)$, as seen in (92) and (93), they may be solved across the entirety of the domain $-\mathcal{L}<x<1$ together with the appropriate boundary conditions on the metallic lithium electrode and the current collector (47),

$$
\left.\mathcal{F}\right|_{x=-\mathcal{L}}=0,\left.\quad \mathcal{F}\right|_{x=1}=0 .
$$

The leading order current density $j(x, t)$, obtained in the preceding asymptotic analysis in (94), may be represented by the following piecewise continuous function:

$$
j(x, t)= \begin{cases}I(t) & x<s_{1}(t), \\ \frac{\mathcal{P} \mathcal{B}(x) \kappa(c)}{\Theta+\mathcal{P B}(x) \kappa(c)}\left(I(t)+2 \Theta \frac{1-t^{+}}{c} \frac{\partial c}{\partial x}\right) & s_{1}(t) \leq x \leq s_{2}(t), \\ 0 & s_{2}(t)<x,\end{cases}
$$

The leading order lithium concentration in the LFP particles obtained from (44), (51), and (61), supplemented with the initial condition (29b), can be summarised as

$$
\frac{\partial c_{c}}{\partial x}=\left\{\left.\begin{array}{ll}
0 & x<s_{1}(t), \\
-\frac{\partial j}{\partial x} & s_{1}(t) \leq x \leq s_{2}(t), \\
0 & s_{2}(t)<x,
\end{array} \quad c_{c}\right|_{t=0}= \begin{cases}1 & x<s_{1}(t), \\
\alpha & s_{1}(t) \leq x \leq s_{2}(t), \\
1 & s_{2}(t)<x .\end{cases}\right.
$$

The positions of the two reaction regions $s_{1}(t)$ and $s_{2}(t)$ are found from the solutions to the following ODEs, which are a result of (77) and (91):

$$
\dot{s}_{1}(t)=\frac{I(t)-\left.j\right|_{x=s_{1}^{+}(t)}}{1-\left.c_{c}\right|_{x=s_{1}^{+}(t)}}, \quad \dot{s}_{2}(t)=-\frac{\left.j\right|_{x=s_{2}^{-}(t)}}{1-\left.c_{c}\right|_{x=s_{2}^{-}(t)}},
$$

subject to the initial conditions $s_{1}(0)=0$ and $s_{2}(0)=1$, and where the superscript plus (minus) notation is used to indicate that the evaluation should be carried out to the right (left) of the discontinuity in $j$ or $c_{c}$. In $\S 6$ we verify the RFM against numerical solutions to the DFN model. 
Higher resistance electrolyte

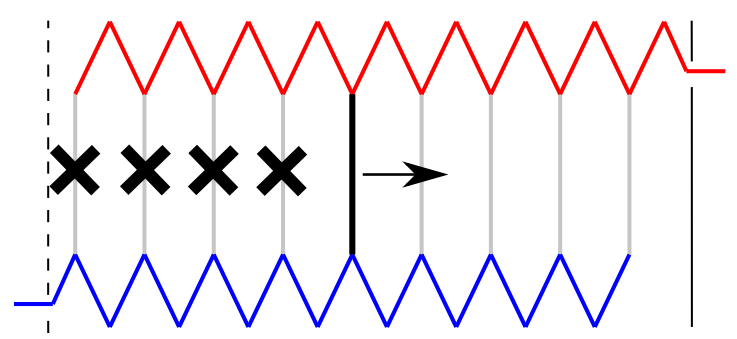

Discharge front starts at separator
Higher resistance solid

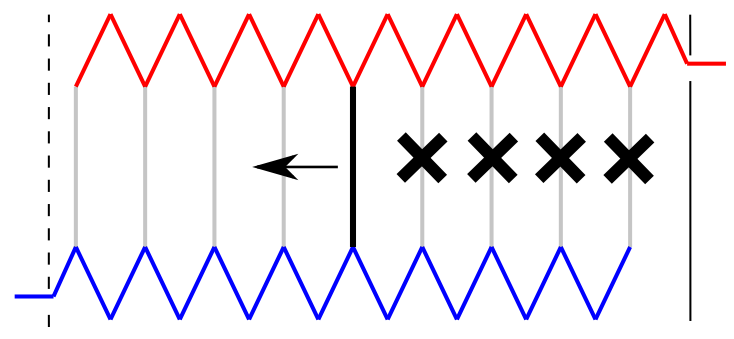

Discharge front starts at current collector

Figure 8: Sketches of a transmission line interpretation of the RFM, where the red and blue resistors represent charge transport pathways in the electrolyte and solid components of the cathode respectively. The vertical black score denotes the position of the reaction front and the arrow indicates its direction of propagation whilst the vertical grey scores indicate the LFP particles. Grey scores with a cross indicate used LFP (unavailable for charge transfer) whilst those without are yet to be used.

\subsection{Interpretation of the reaction front model}

The large value of the dimensionless parameter $\Upsilon$ is responsible for the sharp discharge fronts. However it is important to highlight that, whilst the derivation here is general and accounts for situations where two reaction fronts exist, in typical cathodes only a single discharge front is likely to be observed. If the electrolyte is significantly less conductive than the solid, as is the case in LFP-based cathodes where carbon black can be added to boost the conductivity of the solid phase, only a single discharge front would exist. The intuitive explanation for this is that charge prefers to travel along the path of least resistance. In cathodes with a highly conductive solid, the resistance to ionic motion in the electrolyte is much less than the electronic resistance in the solid and so charge-transfer reaction would occur initially at the separator [43]. This persists until the LFP near the separator has been utilised and the reaction front then begins to propagate towards the current collector, consuming the LFP as it moves. In the scenario where the solid is less conductive than the electrolyte the reaction front initiates at the current collector and subsequently moves toward the separator. Hence, the ratio of conductivities $\Theta / \mathcal{P}=\sigma_{c} / \hat{\mathcal{B}} \hat{\kappa}$ controls the direction of motion of the discharge front(s), and a single front moves from the separator towards the current collector if $\Theta \gg \mathcal{P}$, a single front moves from the current collector towards the separator if $\Theta \ll \mathcal{P}$, and both fronts exist when $\Theta / \mathcal{P}=O(1)$. This idea can be encapsulated as a transmission-line, see Figure 8, where we represents the electrolyte and solid with resistors.

\subsection{Simplification for large C-rates}

We can further simplify the model presented in $\$ 5$ in cases where the current demand is large. We can see from (21) that this corresponds to the values of both $\Theta$ and $\mathcal{P}$ becoming 
small. We see that when $\Theta$ is small the expression for the current (97) collapses as follows:

$$
\text { For } \Theta \ll \mathcal{P}, \quad j= \begin{cases}I(t), & x<s_{2}(t), \\ 0, & x>s_{2}(t),\end{cases}
$$

and correspondingly there is only one reaction front which propagates from the current collector. If, instead, $\mathcal{P}$ is small, then (97) simplifies in the following way:

$$
\text { For } \quad \mathcal{P} \ll \Theta, \quad j= \begin{cases}I(t), & x<s_{1}(t), \\ 0, & x>s_{1}(t),\end{cases}
$$

and the one remaining reaction front intrudes from the separator. It is these simplifications that are relevant to explaining the results discussed in $\S 3$. Finally, we note a simplification that can be exploited if $\Theta$ and $\mathcal{P}$ are comparably small, i.e., to $\Theta \ll 1, \mathcal{P} \ll 1$ but $\Theta / \mathcal{P}=O(1)$. Here, (97) can be written as:

$$
\begin{array}{r}
\text { For } \Theta \ll 1, \quad \mathcal{P} \ll 1, \quad \Theta / \mathcal{P}=O(1) \\
j(x, t)= \begin{cases}I(t) & x<s_{1}(t), \\
\frac{\mathcal{P} \mathcal{B}(x) \kappa(c) I(t)}{\Theta+\mathcal{P B}(x) \kappa(c)} & s_{1}(t) \leq x \leq s_{2}(t), \\
0 & s_{2}(t)<x .\end{cases}
\end{array}
$$

In this case, two reaction fronts still exist simultaneously. It turns out that the RFM obtained by supplementing (95)-(96) and (98)-(99) with either (100), (101) or (102), does not necessarily rely on first taking the limit $\Upsilon \rightarrow \infty$. In fact they can be recovered by performing the analysis for small $\Theta$, small $\mathcal{P}$, or simultaneously small $\Theta$ and $\mathcal{P}$ whilst all other dimensionless parameters are assumed to be of $O(1)$. This asymptotic analysis is presented in $\S$ A.

The case of constant conductivity In cases where the ionic concentration does not vary much in the bulk region, (III), it follows that both the ionic conductivity and current density are almost constant as well. In this case, there is little intercalation in the bulk and (99) can be solved analytically with solution,

$$
s_{1}(t)=\frac{\Theta}{\Theta+\mathcal{P B}(x)} \frac{I(t)}{1-\alpha}, \quad s_{2}(t)=-\frac{\mathcal{P B}(x)}{\Theta+\mathcal{P B}(x)} \frac{I(t)}{1-\alpha} .
$$

\section{Comparison between numerical and asymptotic so- lutions to the model}

We validate the accuracy of the RFM by comparing it with numerical solutions of the full DFN model. The numerical solutions to the DFN model are furnished using the numerical approach outlined in §3.For this purpose we use parameter values that are not necessarily realistic, but rather select them so that the asymptotic structure is clearly visible. We 
validate the full RFM, given by (95)-(99), by setting all dimensionless parameters equal to unity except for $\Upsilon$ which is taken to be 100. The results are shown in figure 9 where we observe that the reduced model is able to accurately reproduce the solution profiles, locations of the discharge fronts, and the cell voltage. A similar validation is carried out for the further simplified RFM, given by (95)-(96) (98)-(99) and (102), by setting all dimensionless parameters equal to unity except $\mathcal{P}$ and $\Theta$ which we take to be 0.01 . In Figure 10 we observe that the simplified model accurately reproduces the solution to the full DFN model. We note the DFN model predicts a surprising feature that the cell voltage exhibits a shortlived initial increase. We have conducted other numerical experiments that reveal that this initial voltage rise is related to our choice of initial condition that particles are at a very low initial SOC, i.e., 3.5\% lithiation see Table 1. At short times, the reaction front is yet to be established and the surface over-potential, $\eta$, required to drive the initial intercalation is relatively high because the prefactor of the Butler-Volmer rate, $\Upsilon c^{1 / 2} c_{c}^{1 / 2}\left(1-c_{c}\right)^{1 / 2}$, i.e. the exchange current density, is small because $c_{c}$ is small, see (26). This gives rise to the initially diminished overall cell voltage. After a short time, $c_{c}$ near the near the edges of the electrode (where the reaction fronts are to be formed) has increased, and so too has the exchange current density, meaning that a decreased surface over-potential is required to drive intercalation and therefore the cell voltage recovers by increasing. This feature is not present in the RFM because our asymptotics is valid for $O(1)$ times after the reaction fronts are fully formed. Returning for a moment to the results in Figures 4-6 and comparing the quality of the agreement with those in Figures 9 and 10 we see that even though the RFM does not reproduce the solution profiles for the electrolyte and solid state lithium concentrations perfectly in realistic cases, it is still able to give a good prediction on the cell voltage. Since this quantity is often the one with the greatest practical importance, the range of operating regimes over which the RFMs can used to predict discharge curves is quite broad. This is made evident through Figure 11, where we plot discharge curves predicted by solutions to both the DFN model and RFM using the values for the modern electrodes are found for C-rates of $1 \mathrm{C}, 10 \mathrm{C}, 20 \mathrm{C}, 50 \mathrm{C}$, and $100 \mathrm{C}$. We note that the discrepancy between the DFN and RFM increases with C-rate, and this can be attributed to the decreasing value of $\Upsilon$ as the current increases.

\section{Conclusions}

We have derived a simplification to the Doyle-Fuller-Newman (DFN) model for the (dis)charge of nano-structured lithium iron phosphate (LFP) cathodes in which it is assumed that the particles are so small that transport inside the electrode particles can be taken to be infinitely fast on the timescales of interest (i.e. that of cell (dis)charge). Numerical solutions to the DFN model in realistic parameter regimes revealed the presence of localised regions of (de)intercalation which propagate from the edges of the electrode into its interior. These reaction fronts form in LFP electrodes much more readily than they can in other common cathode materials (e.g. NMC) because it has a largely flat equilibrium over-potential curve which allows neighbouring electrode particles to be at different states of lithiation without there being a significant potential difference between them. These observations motivated us to carry out an asymptotic analysis of the model and in doing so we were able to derive 

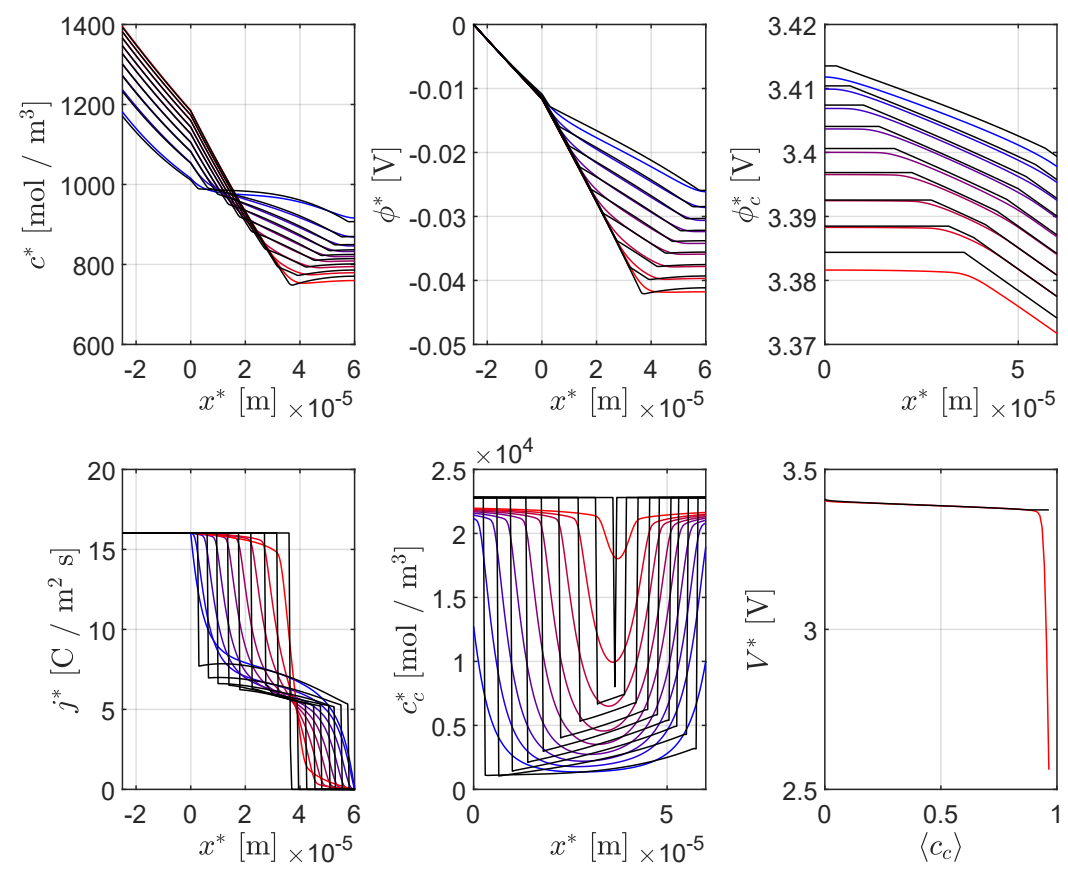

Figure 9: Figure showing the comparison between numerical simulations of the full DFN model (coloured) and the solution predicted by the asymptotic RFM (black). Plots for $c$, $j, \phi, \phi_{c}$, and $c_{c}$ are taken at times $t \in\{0.1,0.2,0.3,0.4,0.5,0.6,0.7,0.8,0.9\}$, as well as the voltage profile $V$. For these plots we take all non-dimensional parameters to be unity with exception that $\Upsilon=100$. 

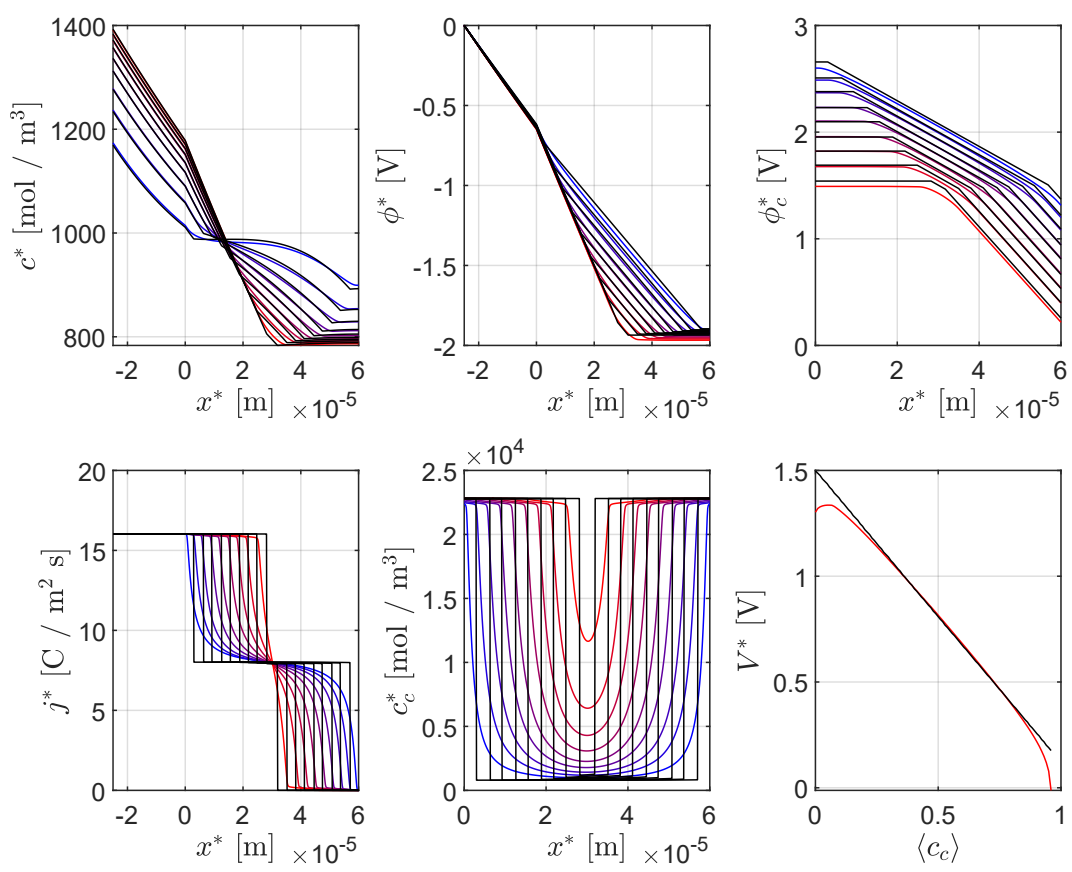

Figure 10: Figure showing the comparison between numerical simulations of the full DFN model (coloured) and the solution predicted by the asymptotic RFM (black). Plots for $c$, $j, \phi, \phi_{c}$, and $c_{c}$ are taken at times $t \in\{0.1,0.2,0.3,0.4,0.5,0.6,0.7,0.8,0.9\}$, as well as the voltage profile $V$. For these plots we take all non-dimensional parameters to be unity with exception that $\Theta=\mathcal{P}=0.01$. 


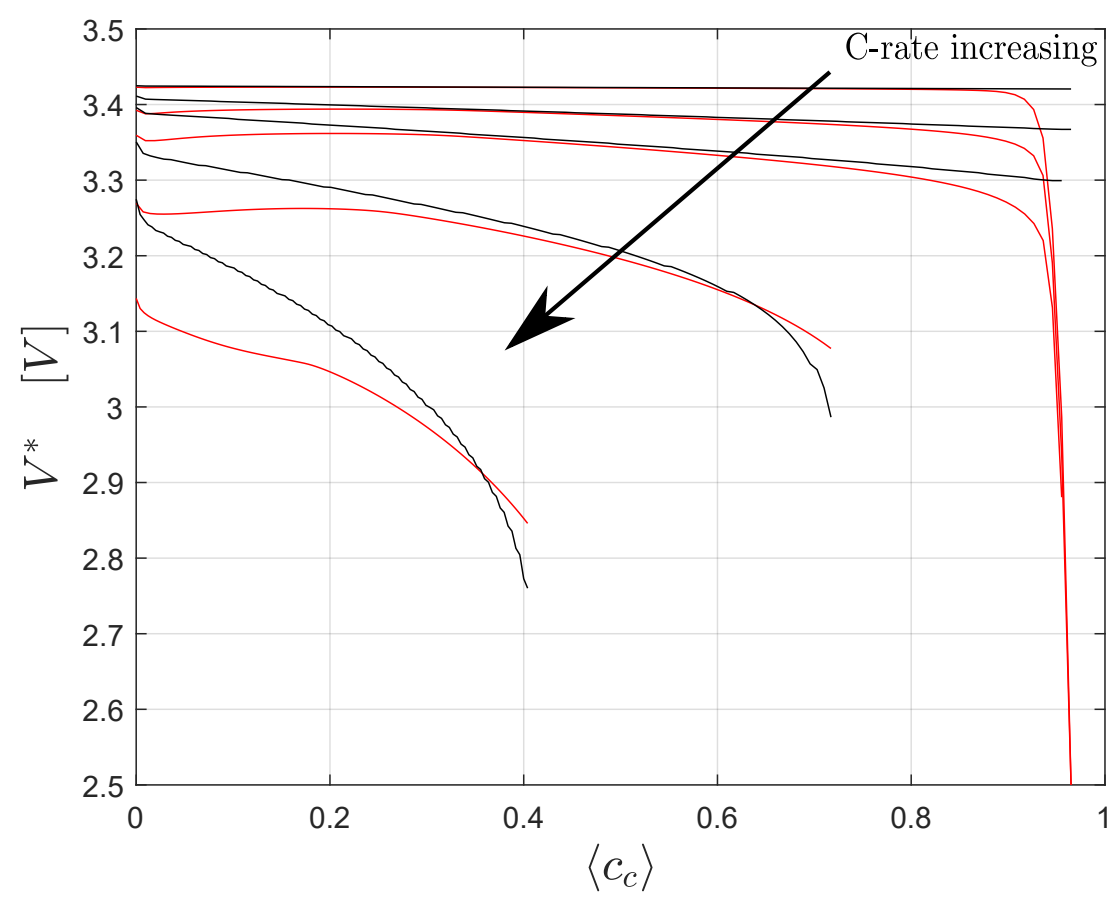

Figure 11: Figure showing the comparison between numerical simulations of the full DFN model (red) and the solution predicted by the asymptotic RFM (black) for modern electrode parameters summarised in table 1 for C-rates of 1, 10, 20, 50, and 100.

a simplified model which we term the reaction front model (RFM). We have shown that the RFM compares favourably to the full DFN model in the parameter regimes outlined above. The RFM can be solved at reduced computational cost and contains only the most important parameters, and so we offer it as an alternative model to the DFN where the cost of simulation needs to be minimised, i.e. in optimisation problems, in parameter estimation studies or for use on the light computational hardware available onboard electric vehicles. Arguably more importantly, the simplicity of the RFM provides insight into the internal dynamics of these devices and can be used to suggests ways in which their design might be improved.

Our analysis demonstrates that tuning the cell parameters affects the speed, direction and sharpness of reaction fronts which are known to exist in electrodes comprised of LFP and other phase change materials (e.g. graphite) [20, 25]. This control opens a promising avenue for improving device design by tuning the conductivities of the electrodes. As just one specific example of where this strategy might be advantageous, consider a cell whose discharge is initially sustainable but that terminates prematurely due to electrolyte depletion near current collector. Here, the rate performance can be improved by (counter-intuitively) decreasing the conductivity of the electrode. This will encourage a discharge front to intrude from the current collector during the early stages of discharge, i.e., when the electrolyte near the current collector remains rich in Li-ions, thereby allowing this previously inaccessible region to be utilised and to contribute towards usable capacity.

It should also be noted that the derivation of the RFM is applicable for all materials with 
flat discharge curves and, whilst LFP is currently the most wide-spread example of this, future development in material science may produce other commercially viable electrode materials with flat OCVs. Moreover, there is an increasing incentive to find alternatives to NMC which is currently the most common cathode material in commercial devices because the supply of cobalt is forecast to be unsustainable [35] and Tesla's recent move to use LFP in the their home storage batteries gives reason to believe that a shift towards LFP might already be underway. The RFM can also be adapted to incorporate the solid transport dynamics, should it be essential to model larger LFP pellet sizes or perhaps more interestingly if the C-rate is so large that even nano-sized LFP particles become rate-limiting.

\section{Acknowledgements}

JMF and GR were supported by the Faraday Institution Multi-Scale Modelling (MSM) project grant number EP/S003053/1. MC was supported by the University of Portsmouth and University Alliance as part of the Doctoral Training Alliance programme.

\section{References}

[1] Amin R. \& Chiang YM., (2016), "Characterization of Electronic and Ionic Transport in $\mathrm{Li}_{1-x} \mathrm{Ni}_{0.33} \mathrm{Mn}_{0.33} \mathrm{Co}_{0.33} \mathrm{O}_{2}\left(\mathrm{NMC}_{333}\right)$ and $\mathrm{Li}_{1-x} \mathrm{Ni}_{0.50} \mathrm{Mi}_{0.20} \mathrm{Co}_{0.30} \mathrm{O}_{2}\left(\mathrm{NMC}_{523}\right)$ as a Function of Li Content". J. Electrochem. Soc., 163(8), A1512-A1517.

[2] Bai P., Cogswell D.A., \& Bazant M.Z., (2011), "Suppression of Phase Separation in $\mathrm{LiFePO}_{4}$ Nanoparticles During Battery Discharge". Nano Lett. 11, 4890-4896.

[3] Bazant, M. Z. (2013). "Theory of chemical kinetics and charge transfer based on nonequilibrium thermodynamics". Accounts of chemical research, 46(5), 1144-1160.

[4] Blomgren, G. (2017), "The Development and Future of Lithium Ion Batteries". J. Electrochem. Soc., 164(1), A5019-A5025.

[5] Bockris, J. O. 'M, Reddy AKN, Gamboa-Aldeco M (2000) "Modern electrochemistry 2A fundamentals of electrodics". Kluwer Academic/Plenum Publishers, Second Edition

[6] Bruggeman D. A. G., Ann. Phys., 24(132), 636 (1935).

[7] B. Kang \& G. Ceder, (2009), "Battery materials for ultrafast charging and discharging". Nature 458, 190-193.

[8] Ciucci F. \& Lai W., (2011), "Derivation of Micro/Macro Lithium Battery Models from Homogenization." Transp. Porous Med. 88(2), 249-270.

[9] Dargaville S. (2013), "Mathematical Modelling of $\mathrm{LiFePO}_{4}$ Cathodes." Queensland University of Technology, $\mathrm{PhD}$ Thesis. 
[10] Dargaville S., Farrell T.W., \& Troy W. (2010), "Predicting active material utilisation in $\mathrm{LiFePO}_{4}$ electrodes using a multi-scale mathematical model." J. Electrochem. Soc., 157(7), A830-A840.

[11] Dargaville S. \& T.W. Farrell (2013), "A comparison of mathematical models for phasechange in high-rate $\mathrm{LiFePO}_{4}$ cathodes". Electrochimica Acta 111, 474-490.

[12] Denis Y., Yu, D., Donoue, K., Inoue, T., Fujimoto, M., \& Fujitani, S. (2006). "Effect of Electrode Parameters on $\mathrm{LiFePO}_{4}$ Cathodes". J. Electrochem. Soc., 153(5), A835.

[13] Doyle M, Fuller T.F., \& and Newman J. (1993), "Modeling of galvanostatic charge and discharge of the lithium-polymer-insertion cell". J. Electrochem. Soc., 140(6), 15261533 .

[14] Ellis B.L., Lee K.T., \& Nazar L.F., (2010), "Positive electrode materials for Li-ion and Li-batteries." Chem. Mater., 22(3), 691-714.

[15] Ender, M., Weber, A., \& Ivers-Tiffée, E. (2013). "A novel method for measuring the effective conductivity and the contact resistance of porous electrodes for lithium-ion batteries". Electrochem. Comms., 34, 130-133.

[16] Farkhondeh, M., Pritzker, M., Fowler, M., \& Delacourt, C. (2017). "Mesoscopic Modeling of a LiFePO4Electrode: Experimental Validation under Continuous and Intermittent Operating Conditions". J. Electrochem. Soc., 164 (11), E3040-E3053.

[17] Farrell, T., Please, C., McElwain, D., \& Swinkels, D. (2000). "Primary Alkaline Battery Cathodes A Three-Scale Model". J. Electrochem. Soc., 147(11), 4034.

[18] Ferguson, T. R., \& Bazant, M. Z. (2014). "Phase transformation dynamics in porous battery electrodes". Electrochimica Acta, 146, 89-97.

[19] Fuller T.F., Doyle M., \& Newman J., (1994), "Simulation and optimisation of the dual lithium ion insertion cell." J. Electrochem. Soc., 141(1), 1-10.

[20] Harris, S.J., Timmons, A., Baker, D.R. \& Monroe, C., (2010). "Direct in situ measurements of Li transport in Li-ion battery negative electrodes". Chemical Physics Letters, 485(4-6), pp.265-274.

[21] Hindermann-Bischoff M. \& Ehrburger-Dolle F., (2001), "Electrical conductivity of carbon black-polyethylene composites Experimental evidence of the change of cluster connectivity in the PTC effect". Carbon, 39, 375-382.

[22] Huang H., Yin SC. \& Nazar L.F., (2001). "Approaching Theoretical Capacity of $\mathrm{LiFePO}_{4}$ at Room Temperature at High Rates". Electrochem. Solid-State Lett. 4, A170A172.

[23] I. Korotkin, G. Richardson \& J. M. Foster, DandyLiion: A fast, and flexible open-source solver for Newman-type models of Li-ion batteries, TBC (2020). 
[24] Joachin H., Kaun T.D., \& Zaghib K., (2009), PrakashaJ., "Electrochemical and Thermal Studies of Carbon-Coated $\mathrm{LiFePO}_{4}$ Cathode." J. Electrochem. Soc., 156(6), A401-A406.

[25] Johns P.A., Roberts M.R., Wakizaka Y., Sanders J.H., \& Owen J.R., (2010), "How the electrolyte limits fast discharge in nanostructured batteries and supercapacitors." Electrochem. Comm., 11(11), 2089-2092.

[26] Jokar A,, Désilets M., Lacroix M., Zaghib K., (2018), "Mesoscopic modeling and parameter estimation of a lithium-ion battery based on $\mathrm{LiFePO}_{4} /$ graphite". J. Power Sources, 379, 84-90.

[27] Julien, C., Mauger, A., Trottier, J., Zaghib, K., Hovington, P., \& Groult, H. (2016). Olivine-Based Blended Compounds as Positive Electrodes for Lithium Batteries. Inorganics, $\mathbf{4}(2), 17$.

[28] Liu, Y., Liu, H., An, L., Zhao, X., \& Liang, G. (2018). Blended spherical lithium iron phosphate cathodes for high energy density lithium-ion batteries. Ionics, 25(1), 61-69. doi: $10.1007 / \mathrm{s} 11581-018-2566-7$

[29] Li Y., Gabaly F.E., Ferguson T.R., Smith R.B., Bartelt N. C., Sugar J.D., Fenton K.R., Cogswell D.A., Jilcoyne A.L.D., Tyliszczak T., Bazant M.Z., \& Chueh W.C., (2014), "Current-induced transition from particle-by-particle to concurrent intercalation in phase-separating battery electrodes." Nature Materials 13, 1149-1156

[30] Malik R., Abdellahi A., \& Ceder G.,(2013), "A Critical Review of the Li Insertion Mechanisms in $\mathrm{LiFePO}_{4}$ Electrodes." J. Electrochem. Soc., 160(5), A3179-A3197.

[31] Malik R., Burch D., Bazant M., \& Ceder G., (2010), "Particle Size Dependence of the Ionic Diffusivity". Nano Lett. 10, 4123-4127

[32] S. G. Marquis, V. Sulzer, R. Timms, C. P. Please, and S. J. Chapman, An asymptotic derivation of a single particle model with electrolyte, arXiv preprint arXiv:1905.12553, (2019)

[33] Mastali, M., Farkhondeh, M., Farhad, S., Fraser, R., \& Fowler, M. (2016). Electrochemical Modeling of Commercial $\mathrm{LiFePO}_{4}$ and Graphite Electrodes: Kinetic and Transport Properties and Their Temperature Dependence. J. Electrochem. Soc., 163(13), A2803A2816.

[34] Ming W. , Jun L. J., Ming H. X., Han W., \& Rong W. C.,(2012), "The effect of local current density on electrode design for lithium-ion batteries." J. Power Sources, 207, $127-133$.

[35] Nkulu, C.B.L., Casas, L., Haufroid, V., De Putter, T., Saenen, N.D., Kayembe-Kitenge, T., Obadia, P.M., Mukoma, D.K.W., Ilunga, J.M.L., Nawrot, T.S. \& Numbi, O.L., (2018). "Sustainability of artisanal mining of cobalt in DR Congo". Nature sustainability, 1(9), pp.495-504. 
[36] Park M., Zhang X., Chung M, Less GB, \& Sastry A.M. (2010), "A review of conduction phenomena in Li-ion batteries". J. Power Sources.,

[37] Padhi A.K., Nanjundaswamy K.S., \& Goodenough J.B., (1997), "Phospho-olivines as positive electrode materials for rechargeable lithium batteries". J. Electrochem. Soc., 144(4), 1188-1194.

[38] Patel, K., Paulsen, J., \& Desilvestro, J. (2003). Numerical simulation of porous networks in relation to battery electrodes and separators. J. Power Sources, 122(2), 144-152.

[39] Phillip J.A., (2011), "Investigations of Rate Limitation in Nanostructured Composite Electrodes and Experiments Towards a 3D Li-ion Microbattery", Uni. of Southampton, $\mathrm{PhD}$ Thesis.

[40] Prada E., Domenico D.D., Creff Y., Bernard J., Sauvant-Moynot V., Huet F., (2012), "Simplified electrochemical and thermal model of $\mathrm{LiFePO}_{4}$-graphite Li-Ion batteries for fast charge applications". J. Electrochem. Soc. 159: A1508-A1519.

[41] Prosini P.P., Lisi M., Zane D., \& Pasquali M., (2002), "Determination of the chemical diffusion coefficient of lithium in $\mathrm{LiFePO}_{4}$." Solid State Ionics, 148(1-2), 45-51.

[42] Rajabloo, B., Jokar, A., Wakem, W., Désilets, M., \& Brisard, G. (2018). Lithium iron phosphate electrode semi-empirical performance model. J. Appl. Electrochem., 48 (6), 663-674.

[43] Ranom R. (2015), "Mathematical modelling of lithium-ion batteries". Uni. of Southampton, $\mathrm{PhD}$ Thesis.

[44] Richardson G., Denuault G., \& Please C.P., (2012). "Multiscale modelling and analysis of lithium-ion battery charge and discharge." J. Eng. Math. 72(1), 41-72.

[45] G. Richardson, I. Korotkin, R. Ranom, M. Castle and J.M. Foster, "Generalised single particle models for high-rate operation of graded lithium-ion electrodes: systematic derivation and validation", arXiv preprint, arXiv :1907.09410,

[46] Safari, M., \& Delacourt, C. (2011). Mathematical Modeling of Lithium Iron Phosphate Electrode: Galvanostatic Charge/Discharge and Path Dependence. J. Electrochem. Soc., 158(2), A63.

[47] Saroha, R., Panwar, A., Gaur, A., Sharma, Y., Kumar, V., \& Tyagi, P. (2018). Electrochemical studies of novel olivine-layered (LiFePO4-Li2MnO3) dual composite as an alternative cathode material for lithium-ion batteries. J. Electrochem. Soc., 22(8), 25072513.

[48] Srinivasan V. \& Newman J., (2004), "Design and optimization of a natural graphite/iron phosphate Lithium-ion cell". J. Electrochem. Soc., 151(10), A1530-A1538.

[49] Srinivasan V. \& Newman J., (2004), "Discharge model for the lithium iron-phosphate electrode". J. Electrochem. Soc., 151(10). A1517-A1529. 
[50] Stewart, S., \& Newman, J. (2008). Measuring the Salt Activity Coefficient in LithiumBattery Electrolytes. J. Electrochem. Soc. 155 (6), A458.

[51] Stewart, S., Albertus, P., Srinivasan, V., Plitz, I., Pereira, N., Amatucci, G., \& Newman, J. (2008). Optimizing the Performance of Lithium Titanate Spinel Paired with Activated Carbon or Iron Phosphate. J. Electrochem. Soc., 155 (3), A253.

[52] Tesla (2020). Annual Shareholder Meeting and Battery Day. Tesla, Inc. 901 Page Ave. Fremont, CA 94538. September 22.

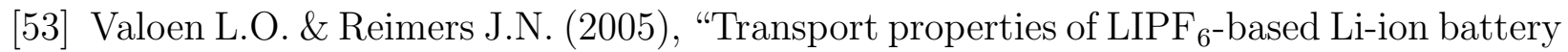
electrolytes". J. Electrochem. Soc., 152(5), A882-A891.

[54] Wang G.X., Bewlay S.L., Konstantinova K., Liu H.K., Dou S.X., \& Ahn J.-H., (2004), "Physical and electrochemical properties of doped lithium iron phosphate electrodes". Electrochimica Acta, 50, 443-447.

[55] Wang, M., Li, J., He, X., Wu, H., \& Wan, C. (2012). "The effect of local current density on electrode design for lithium-ion batteries". J. Power Sources, 207, 127-133.

[56] Zheng H., Li J., Song X., Liu G., \& Battaglia V.S., (2012), "A comprehensive understanding of electrode thickness effects on the electrochemical performances of Li-ion battery cathodes". Electrochim. Acta 71, 258-265.

[57] https://patentimages.storage.googleapis.com/aa/bb/b0/e29140f2b7e47b/US6921608.pdf

\section{Appendices}

\section{A Asymptotic analysis}

The computations shown in $\S 6$, namely figure 10 , suggest that an asymptotic solution relevant to high C-rates is available based on small values of $\mathcal{P}$ and $\Theta$. The solution is characterised by a wide region of slowly reacting electrode particles in the bulk separated from regions of fully lithiated particles by narrow rapidly reacting regions which intrude from the edges of the electrode into its interior as discharge proceeds. Formally, we shall consider the limit in which both $\mathcal{P} \rightarrow 0$ and $\Theta \rightarrow 0$, whilst we take all other parameters to be $O(1)$. The dynamics of both limits, taken singularly and simultaneously, can be examined by letting $\Theta / \mathcal{P}=\hat{\mathcal{P}}$. Under this transformation of parameters, we eliminate $\mathcal{P}$ from the non-dimensional DFN model and reduce the model by taking $\Theta \rightarrow 0$ and $\hat{\mathcal{P}}=O(1)$. The solution is described by seven asymptotic regions which we will distinguish using Roman numeral superscripts as follows: the central slowly reacting region (denoted region $(I V)$ ), the two reacted regions near the separator and current collector (termed regions $(I)$ and (VII) respectively), two narrow moving reaction-initiation fronts (regions $(I I I)$ and $(V)$ ), and two narrow moving reaction fronts (regions $(I I)$ and $(V I)$ ). 


\section{A.1 Outer regions}

The outer regions, namely region $(I)$ where $-\mathcal{L}<x<s_{1}(t)$, region $(I V)$ where $s_{1}(t)<$ $x<s_{2}(t)$, and region $(V I I)$ where $s_{2}(t)<x<1$, are analysed on the length-scale of the electrode.

Region $(I)$ : reacted region adjacent to separator In region $(I)$, where $-\mathcal{L}<x<$ $s_{1}(t)$, the particles are almost fully lithiated and consequently it is unable to sustain a significant reaction or convert a significant portion of the ionic current (which enters the electrode via the adjacent separator) into electronic form. It follows, from (31), that the electronic current in this region is small and thus the potential gradient across the electrode particles must also be significantly smaller than that across the electrolyte in this region. The asymptotic solution in this region is found by expanding as follows:

$$
\begin{aligned}
c^{(I)} & =c_{0}^{(I)}(x, t)+\cdots & & \mathcal{F}^{(I)}=\mathcal{F}_{0}^{(I)}(x, t)+\cdots \\
j^{(I)} & =I(t)+\cdots & & j_{c}^{(I)}=0+\cdots \\
\phi^{(I)} & =\Theta^{-1} \phi_{-1}^{(I)}(x, t)+\cdots & & \phi_{c}^{(I)}=\Theta^{-1} \phi_{c,-1}^{(I)}(t)+\cdots \\
c_{c}^{(I)} & =1+\cdots & & G^{(I)}=0+\cdots .
\end{aligned}
$$

Here we use the superscript $(I)$ to indicate variables within this region. Equations (24a), (25), and (26) are automatically satisfied at leading order by the expansions (105)-(108). The three remaining leading order variables $c_{0}^{(I)}, \mathcal{F}_{0}^{(I)}$ and $\phi_{-1}^{(I)}$ satisfy equations that are obtained by balancing the leading order terms in (23) and (24b); these read:

$$
\begin{array}{ll}
\mathcal{N} \frac{\partial c_{0}^{(I)}}{\partial t}+\frac{\partial \mathcal{F}_{0}^{(I)}}{\partial x}=0, & \mathcal{F}_{0}^{(I)}=-\mathcal{B}(x) D\left(c_{0}^{(I)}\right) \frac{\partial c_{0}^{(I)}}{\partial x}-\Gamma\left(1-t^{+}\right) I(t), \\
I(t)=-\frac{\mathcal{B}(x) \kappa\left(c_{0}^{(I)}\right)}{\hat{\mathcal{P}}} \frac{\partial \phi_{-1}^{(I)}}{\partial x} &
\end{array}
$$

and satisfy the boundary condition

$$
\left.\mathcal{F}_{0}^{(I)}\right|_{x=-\mathcal{L}}=0,\left.\quad \phi_{-1}^{(I)}\right|_{x=-\mathcal{L}}=0,
$$

on the separator (obtained from (27b) at leading order).

Region (VII): reacted region adjacent to current collector In the other reacted region, lying in $s_{2}(t)<x<1$, the asymptotic expansion proceeds exactly as it does in region $(I)$ (for similar reasons) with the notable exception that the current flows almost entirely in the solid electrode matrix (rather than the electrolyte); it reads:

$$
\begin{aligned}
& c^{(V I I)}=c_{0}^{(V I I)}(x, t)+\cdots \quad \mathcal{F}^{(V I I)}=\mathcal{F}_{0}^{(V I I)}(x, t)+\cdots \\
& j^{(V I I)}=0+\cdots \quad j_{c}^{(V I I)}=I(t)+\cdots \\
& \phi^{(V I I)}=\Theta^{-1} \phi_{-1}^{(V I I)}(t)+\cdots \quad \phi_{c}^{(V I I)}=\Theta^{-1} \phi_{-1}^{(V I I)}(x, t)+\cdots \\
& c_{c}^{(V I I)}=1+\cdots \quad G^{(V I I)}=0+\cdots
\end{aligned}
$$


Equations (24), (25a), (25c), and (26) are automatically satisfied at leading order by the expansions (112)-(115). The remaining leading order variables $c_{0}^{(I I)}, \mathcal{F}_{0}^{(I I)}$ and $\phi_{c,-1}^{(I I)}$ satisfy the leading balances in equations (23) and $(25 \mathrm{~b})$ which read:

$$
\begin{aligned}
& \mathcal{N} \frac{\partial c_{0}^{(V I I)}}{\partial t}+\frac{\partial \mathcal{F}_{0}^{(V I I)}}{\partial x}=0, \quad \mathcal{F}_{0}^{(V I I)}=-\mathcal{B}(x) D\left(c_{0}^{(V I I)}\right) \frac{\partial c_{0}^{(V I I)}}{\partial x} \\
& -I(t)=\frac{\partial \phi_{c,-1}^{(V I I)}}{\partial x} .
\end{aligned}
$$

and satisfy the boundary condition

$$
\left.\mathcal{F}_{0}^{(V I I)}\right|_{x=1}=0,
$$

on the current collector (obtained from (28a) at leading order)

Region $(I V)$ : central slowly reacting region In the central slowly reacting region, lying in $s_{1}(t)<x<s_{2}(t)$, there are significant current flows in both the electrolyte and the solid and significant charge transfer is occurring, i.e. there is an $O(1)$ amount of reaction in this region. Since the electrode particles here still have space to accommodate more $\mathrm{Li}^{+}$, the only way in which (26) is consistent with $O(1)$ reaction rates is if the potentials in the electrolyte and solid are almost equal. The asymptotic solution can be found by expanding as follows

$$
\begin{array}{lll}
c^{(I V)}=c_{0}^{(I V)}(x, t)+\cdots & \mathcal{F}=\mathcal{F}_{0}^{(I V)}(x, t)+\cdots \\
j^{(I V)}=j_{0}^{(I V)}(x, t)+\cdots & & j_{c}^{(I V)}=I(t)-j_{0}^{(I V)}(x, t)+\cdots \\
\phi^{(I V)}=\Theta^{-1} \phi_{-1}^{(I V)}(x, t)+\cdots & & \phi_{c}^{(I V)}=\Theta^{-1} \phi_{-1}^{(I V)}(x, t)+\cdots \\
c_{c}^{(I V)}=c_{c, 0}^{(I V)}(x, t)+\cdots & & G^{(I V)}=G_{0}^{(I V)}(x)+\cdots
\end{array}
$$

On substituting this expansion into (23)-(26) we find that the leading order variables $c_{0}^{(I V)}$, $\mathcal{F}_{0}^{(I V)}, j_{0}^{(I V)}, \phi_{-1}^{(I V)}$ and $G_{0}^{(I V)}$ satisfy the following equations

$$
\begin{array}{ll}
\mathcal{N} \frac{\partial c_{0}^{(I V)}}{\partial t}+\frac{\partial \mathcal{F}_{0}^{(I V)}}{\partial x}=0, & \mathcal{F}_{0}^{(I V)}=-\mathcal{B}(x) D\left(c_{0}^{(I V)}\right) \frac{\partial c_{0}^{(I V)}}{\partial x}-\Gamma\left(1-t^{+}\right) j_{0}^{(I V)}, \\
j_{0}^{(I V)}=-\frac{\mathcal{B}(x) \kappa\left(c_{0}^{(I V)}\right)}{\hat{\mathcal{P}}} \frac{\partial \phi_{-1}^{(I V)}}{\partial x}, & \frac{\partial \phi_{-1}^{(I V)}}{\partial x}=j_{0}^{(I V)}-I(t), \\
\frac{d c_{c, 0}^{(I V)}}{d t}=-G_{0}^{(I V)}, & \frac{\partial j_{0}^{(I V)}}{\partial x}=G_{0}^{(I V)},
\end{array}
$$

By eliminating the leading order potential gradient between equations (124a) and (124b) we can solve for the ionic current in terms of the leading order electrolyte concentrations, yielding

$$
j_{0}^{(I V)}=I(t) \frac{\mathcal{B}(x) \kappa\left(c_{0}^{(I V)}\right)}{\mathcal{B}(x) \kappa\left(c_{0}^{(I V)}\right)+\hat{\mathcal{P}}} .
$$


We can then solve for the lithium content in the solid in terms of the ionic current from equation (125) by eliminating $G_{0}^{(I V)}$, integrating with respect to time $t$ and supplying the initial condition (12b), giving:

$$
c_{c, 0}^{(I V)}=\alpha-\int_{0}^{t} \frac{\partial j_{0}^{(I V)}}{\partial x} d t^{\prime} .
$$

\section{A.2 Regions around left-hand reaction front on $x=s_{1}(t)$}

We now investigate the narrow reaction regions separating the three outer regions discussed above. In addition to the spatial length-scale of the outer regions discussion in $\S$ A.1 there are two further length-scales to consider. The analysis of regions $(I I I)$ and $(V)$ is performed under the scale of $O\left(\Theta^{1 / 2}\right)$, whilst that of regions $(I I)$ and $(V I)$ is chosen to be $O(\Theta)$.

Region $(I I I)$ : left-hand reaction initiation region The left-hand reaction initiation region, located about $x=s_{1}(t)$, has width $O\left(\Theta^{1 / 2}\right)$ and is therefore studied under a change of coordinates given by

$$
x-s_{1}(t)=\Theta^{1 / 2} z,
$$

and so the local equations become

$$
\begin{aligned}
& -\dot{s}_{1}(t) \mathcal{N} \frac{\partial c^{(I I)}}{\partial z}+\Theta^{1 / 2} \mathcal{N} \frac{\partial c^{(I I I)}}{\partial t}+\frac{\partial \mathcal{F}^{(I I I)}}{\partial z}=0, \\
& \mathcal{F}^{(I I I)}=-\Theta^{-1 / 2} \mathcal{B}(x) D\left(c^{(I I I)}\right) \frac{\partial c^{(I I I)}}{\partial z}-\Gamma\left(1-t^{+}\right) j^{(I I I)}, \\
& \frac{\partial j^{(I I I)}}{\partial z}=\Theta^{1 / 2} G^{(I I I)} \\
& j^{(I I I)}=-\Theta^{1 / 2} \frac{\mathcal{B}(x) \kappa\left(c^{(I I I)}\right)}{\hat{\mathcal{P}}}\left(\frac{\partial \phi^{(I I I)}}{\partial z}-2 \frac{1-t^{+}}{c^{(I I I)}} \frac{\partial c^{(I I I)}}{\partial z}\right), \\
& \frac{\partial j_{c}^{(I I I)}}{\partial z}=-\Theta^{1 / 2} G^{(I I I)} \\
& j_{c}^{(I I I)}=-\Theta^{1 / 2} \frac{\partial \phi_{c}^{(I I I)}}{\partial z}, \\
& -\Theta^{-1 / 2} \dot{s}_{1}(t) \frac{\partial c_{c}^{(I I I)}}{\partial z}+\frac{\partial c_{c}^{(I I I)}}{\partial t}=-G^{(I I I)}, \\
& G=\Upsilon \sqrt{c^{(I I I)} c_{c}^{(I I I)}\left(1-c_{c}^{(I I I)}\right)}\left(e^{\frac{\eta}{2}}-e^{-\frac{\eta}{2}}\right), \\
& \eta=\phi_{c}^{(I I I)}-\phi^{(I I I)} \text {. }
\end{aligned}
$$

The asymptotic solution can then be found by expanding as follows

$$
\begin{aligned}
c^{(I I I)} & =c_{0}^{(I I I)}(t)+\cdots, & \mathcal{F}^{(I I I)} & =\mathcal{F}_{0}^{(I I I)}(t)+\cdots, \\
j^{(I I I)} & =j_{0}^{(I I I)}(t)+\cdots, & \phi^{(I I I)} & =\Theta^{-1} \phi_{-1}^{(I I I)}(t)+\phi_{0}^{(I I I)}(z, t)+\cdots, \\
j_{c}^{(I I I)} & =I(t)-j_{0}^{(I I I)}(t)+\cdots, & \phi_{c}^{(I I I)} & =\Theta^{-1} \phi_{-1}^{(I I I)}(t)+\phi_{c, 0}^{(I I I)}(z, t)+\cdots, \\
c_{c}^{(I I I)} & =c_{c, 0}^{(I I I)}(t)+\cdots, & G^{(I I I)} & =G_{0}^{(I I I)}(z, t)+\cdots,
\end{aligned}
$$


which, by design, satisfy equations (129)-(137). The $O(1)$ variables for the ionic and electronic potentials $\phi_{0}^{(I I I)}$ and $\phi_{c, 0}^{(I I I)}$ are to be solved. A closed equation for these variables can be found by substituting equations (132) and (134) into equations (131) and (133) respectively, adding the two and substituting the expansions into the resulting equation we have

$$
\frac{\partial^{2}}{\partial z^{2}}\left(\phi_{c, 0}^{(I I I)}-\phi_{0}^{(I I I)}\right)=\hat{K}(t) \sinh \left(\frac{\phi_{c, 0}^{(I I I)}-\phi_{0}^{(I I I)}}{2}\right)
$$

where

$$
\hat{K}(t)=2 \Upsilon \frac{\mathcal{B}(x) \kappa\left(c_{0}^{(I I I)}(t)\right)+\hat{\mathcal{P}}}{\mathcal{B}(x) \kappa\left(c_{0}^{(I I I)}(t)\right)}\left(c_{0}^{(I I I)}(t)\right)^{1 / 2}\left(c_{c, 0}^{(I I I)}(t)\right)^{1 / 2}\left(1-c_{c, 0}^{(I I I)}(t)\right)^{1 / 2} .
$$

Equation (142) can be solved analytically, giving

$$
\phi_{c, 0}^{(I I I)}-\phi_{0}^{(I I I)}=\hat{\phi}(t) \pm 4 \log \left(\operatorname{coth}\left(\sqrt{\frac{\hat{K}(t)}{4}}\left(z-z_{0}(t)\right)\right)\right)
$$

where $z_{0}(t)$ and $\hat{\phi}(t)$ are constants of integration, which will be determined by matching to the adjacent regions. Performing this matching will require the limiting behaviour of this solution, which are

$$
\begin{array}{r}
\phi_{c, 0}^{(I I I)}-\phi_{0}^{(I I I)} \rightarrow \hat{\phi}(t) \quad \text { as } \quad z \rightarrow \infty, \\
\phi_{c, 0}^{(I I I)}-\phi_{0}^{(I I I)} \sim 4 \log \left(\sqrt{\frac{\hat{K}}{4}}\left(z_{0}-z\right)\right) \quad \text { as } \quad z \nearrow z_{0} .
\end{array}
$$

Region $(I I)$ : left-hand main reaction region The left-hand reaction region, located about $x=s_{1}(t)$, has width $O(\Theta)$ and is therefore studied under a change of coordinates given by

$$
z_{0}-z=\Theta^{1 / 2} y
$$

and so the local equations become

$$
\begin{gathered}
\mathcal{N}\left(-\Theta^{-1} \dot{s}_{1}(t) \frac{\partial c^{(I I)}}{\partial y}+\frac{\partial c^{(I I)}}{\partial t}\right)+\Theta^{-1} \frac{\partial \mathcal{F}^{(I I)}}{\partial y}=0 \\
\mathcal{F}^{(I I)}=-\Theta^{-1} \mathcal{B}(x) D\left(c^{(I I)}\right) \frac{\partial c^{(I I)}}{\partial y}-\Gamma\left(1-t^{+}\right) j^{(I I)} \\
\Theta^{-1} \frac{\partial j^{(I I)}}{\partial y}=G^{(I I)} \\
j^{(I I)}=-\frac{\mathcal{B}(x) \kappa\left(c^{(I I)}\right)}{\hat{\mathcal{P}}}\left(\frac{\partial \phi^{(I I)}}{\partial y}-2 \frac{1-t^{+}}{c^{(I I)}} \frac{\partial c^{(I I)}}{\partial y}\right)
\end{gathered}
$$




$$
\begin{gathered}
\Theta^{-1} \frac{\partial j_{c}^{(I I)}}{\partial y}=-G^{(I I)} \\
j_{c}^{(I I)}=-\frac{\partial \phi_{c}^{(I I)}}{\partial y} \\
-\Theta^{-1} \dot{s}_{1}(t) \frac{\partial c_{c}^{(I I)}}{\partial y}+\frac{\partial c_{c}^{(I I)}}{\partial t}=-G^{(I I)}, \\
G=\Upsilon \sqrt{c^{(I I)} c_{c}^{(I I)}\left(1-c_{c}^{(I I)}\right)}\left(e^{\frac{\eta}{2}}-e^{-\frac{\eta}{2}}\right), \\
\eta=\phi_{c}^{(I I)}-\phi^{(I I)} .
\end{gathered}
$$

The asymptotic solution can then be found by expanding as follows

$$
\begin{aligned}
c^{(I I)} & =c_{0}^{(I I)}(t)+\cdots, & \mathcal{F}^{(I I)} & =\mathcal{F}_{0}^{(I I)}(t)+\cdots, \\
j^{(I I)} & =j_{0}^{(I I)}(y, t)+\cdots, & & \phi^{(I I)}=\Theta^{-1} \phi_{-1}^{(I I)}(t)+2 \log (\Theta)+\phi_{0}^{(I I)}(y, t)+\cdots, \\
j_{c}^{(I I)} & =I(t)-j_{0}^{(I I)}(y, t)+\cdots, & \phi_{c}^{(I I)} & =\Theta^{-1} \phi_{-1}^{(I I)}(t)+\phi_{c, 0}^{(I I)}(y, t)+\cdots \\
c_{c}^{(I I)} & =c_{c, 0}^{(I I)}(y, t)+\cdots, & & G^{(I I)}=\Theta^{-1} G_{-1}^{(I I)}(y, t)+\cdots,
\end{aligned}
$$

which, by design, satisfy equations (148)-(149). Upon substituting equation (151) into (150) and substituting equation (152) into (151) we find that, by substitution of the expansions (157)-(160), the first order variables $c_{c, 0}^{(I I)}$ and $j_{0}^{(I I)}$, as well as the second order variables $\phi_{0}^{(I I)}$ and $\phi_{c, 0}^{(I I)}$, satisfy the following equations:

$$
\begin{array}{r}
\frac{\partial^{2}}{\partial y^{2}}\left(\phi_{c, 0}^{(I I)}-\phi_{0}^{(I I)}\right)=\frac{\mathcal{B}(x) \kappa\left(c_{0}^{(I I)}(t)\right)+\hat{\mathcal{P}}}{\mathcal{B}(x) \kappa\left(c_{0}^{(I I)}(t)\right)} G_{-1}^{(I I)}, \\
-\dot{s}_{1}(t) \frac{\partial c_{c, 0}^{(I I)}}{\partial y}=G_{-1}^{(I I)}, \quad \frac{\partial j_{0}^{(I I)}}{\partial y}=-\dot{s}_{1}(t) \frac{\partial c_{c, 0}^{(I I)}}{\partial y} \\
G_{-1}^{(I I)}=\Upsilon\left(c_{0}^{(I I)}(t)\right)^{1 / 2}\left(c_{c, 0}^{(I I)}\right)^{1 / 2}\left(1-c_{c, 0}^{(I I)}\right)^{1 / 2} \exp \left(\frac{\phi_{c, 0}^{(I I)}-\phi_{0}^{(I I)}}{2}\right) .
\end{array}
$$

This pair of equations for $\phi_{c, 0}^{(I I)}-\phi_{0}^{(I I)}$ and $c_{c, 0}^{(I I)}$ are to be solved subject to matching conditions from the neighbouring regions, namely $(I)$ and $(I I I)$.

$$
\begin{gathered}
\phi_{c, 0}^{(I I)}-\phi_{0}^{(I I)} \sim 4 \log \left(-\sqrt{\frac{\hat{K}}{4}} y\right) \text { as } y \rightarrow \infty \\
\left.\begin{array}{c}
\frac{\partial}{\partial w}\left(\phi_{c, 0}^{(I I)}-\phi_{0}^{(I I)}\right) \sim I(t) \\
c_{c, 0}^{(I I)} \sim 1
\end{array}\right\} \text { as } y \rightarrow-\infty
\end{gathered}
$$


The limiting behaviour (146) can be rewritten in the spatial variable of region (II), see (147), as

$$
2 \log \Theta+O(1) \sim \phi_{c, 0}^{(I I I)}-\phi_{0}^{(I I I)}
$$

and for matching this requires (164a). Matching the concentration in the solid leads to the condition (164c) whilst the current requires

$$
j_{0}^{(I I)}-j_{c, 0}^{(I I)} \rightarrow I(t) \text { as } y \rightarrow-\infty .
$$

Matching regions $(I)-(I V)$ across left reaction front near $x=s_{1}(t) \quad$ Upon matching the leading order lithium concentrations and fluxes (in the electrolyte) across the regions adjacent to the left-hand reaction front situated about $x=s_{1}(t)$ we find that

$$
\begin{gathered}
\left.c_{0}^{(I)}\right|_{x \nearrow s_{1}(t)}=c_{0}^{(I I)}(t)=c_{0}^{(I I I)}(t)=\left.c_{0}^{(I V)}\right|_{x \searrow s_{1}(t)}, \\
\left.\mathcal{F}_{0}^{(I)}\right|_{x \nearrow s_{1}(t)}=\mathcal{F}_{0}^{(I I)}(t)=\mathcal{F}_{0}^{(I I I)}(t)=\left.\mathcal{F}_{0}^{(I V)}\right|_{x \searrow s_{1}(t)} .
\end{gathered}
$$

The equations for the concentration and flux of lithium in the electrolyte however cannot be closed at this stage; this will be done in $\S$ A.4 after matching across the right-hand reaction front around $x=s_{2}(t)$ in $\S$ A.3. Performing a similar operation for the leading order electrolyte potentials we obtain the following matching conditions:

$$
\left.\phi_{-1}^{(I)}\right|_{x \nearrow s_{1}(t)}=\phi_{-1}^{(I I)}(t)=\phi_{-1}^{(I I I)}(t)=\left.\phi_{-1}^{(I V)}\right|_{x \searrow s_{1}(t)} .
$$

On matching the leading order electronic potentials across regions $(I)$ and $(I I)$ we find

$$
\phi_{c,-1}^{(I)}(t)=\phi_{c,-1}^{(I I)}(t) .
$$

The $O(1)$ terms for the difference between the ionic and electronic potentials must be matched between regions $(I I I)$ and $(I V)$. This leads to the requirement that

$$
\hat{\phi}(t)=\left.\phi_{c, 0}^{(I V)}\right|_{x \searrow s_{1}(t)}-\left.\phi_{0}^{(I V)}\right|_{x \searrow s_{1}(t)} \cdot
$$

Matching the leading order lithium content inside the electrode particles across all four regions leads to the conditions

$$
\left.c_{c, 0}^{(I I)}\right|_{y \rightarrow-\infty}=1,\left.\quad c_{c, 0}^{(I I)}\right|_{y \rightarrow \infty}=c_{c, 0}^{(I I I)}(t)=\left.c_{c, 0}^{(I V)}\right|_{x \searrow s_{1}(t)},
$$

Finally, the matching conditions on the leading order ionic current densities $j$ are

$$
\left.j_{0}^{(I I)}\right|_{y \rightarrow-\infty}=I(t),\left.\quad j_{0}^{(I I)}\right|_{y \rightarrow \infty}=j_{0}^{(I I I)}(t)=\left.j_{0}^{(I V)}\right|_{x \searrow s_{1}(t)} .
$$

The position of the first reaction layer $s_{1}(t)$ is found by integrating equation (162.b) and applying conditions (173)-(174), giving

$$
\dot{s}_{1}(t)=\frac{\left.j_{0}^{(I I)}\right|_{y \rightarrow \infty}-\left.j_{0}^{(I I)}\right|_{y \rightarrow-\infty}}{\left.c_{c, 0}^{(I I)}\right|_{y \rightarrow \infty}-\left.c_{c, 0}^{(I I)}\right|_{y \rightarrow-\infty}}=\frac{I(t)-j_{0}^{(I I I)}(t)}{1-c_{c, 0}^{(I I I)}(t)}
$$

with the initial condition $\left.s_{1}(t)\right|_{t=0}=0$. 


\section{A.3 Regions around right-hand reaction front on $x=s_{2}(t)$}

Much as in $\S \mathrm{A} .2$ the two inner regions $(V)$ and $(V I)$, located around the reaction front at $x=s_{2}(t)$, are analysed on separate length-scales. The analysis of region $(V)$ occurs on the $O\left(\Theta^{1 / 2}\right)$ length-scale whilst that of regions $(V I)$ is occurs on the $O(\Theta)$ length-scale.

Regions $(V)$ : right-hand reaction initiation region The right-hand reaction initiation region, located about $x=s_{2}(t)$, has width $O\left(\Theta^{1 / 2}\right)$ and is therefore studied under a change of coordinates given by

$$
x-s_{2}(t)=\Theta^{1 / 2} v
$$

and so the local equations become

$$
\begin{aligned}
& -\Theta^{-1 / 2} \dot{s}_{2}(t) \mathcal{N} \frac{\partial c^{(V)}}{\partial v}+\mathcal{N} \frac{\partial c^{(V)}}{\partial t}+\Theta^{-1 / 2} \frac{\partial \mathcal{F}^{(V)}}{\partial v}=0 \\
& \mathcal{F}^{(V)}=-\Theta^{-1 / 2} \mathcal{B}(x) D\left(c^{(V)}\right) \frac{\partial c^{(V)}}{\partial v}-\Gamma\left(1-t^{+}\right) j^{(V)} \\
& \Theta^{-1 / 2} \frac{\partial j}{\partial v}=G^{(V)} \\
& j^{(V)}=-\Theta^{1 / 2} \frac{\mathcal{B}(x) \kappa\left(c^{(V)}\right)}{\hat{\mathcal{P}}}\left(\frac{\partial \phi^{(V)}}{\partial z}-2 \frac{1-t^{+}}{c^{(V)}} \frac{\partial c^{(V)}}{\partial v}\right), \\
& \Theta^{-1 / 2} \frac{\partial j_{c}^{(V)}}{\partial v}=-G^{(V)}, \\
& j_{c}^{(V)}=-\Theta^{1 / 2} \frac{\partial \phi_{c}^{(V)}}{\partial v}, \\
& -\Theta^{-1 / 2} \dot{s}_{2}(t) \frac{\partial c_{c}^{(V)}}{\partial v}+\frac{\partial c_{c}^{(V)}}{\partial t}=-G^{(V)}, \\
& G^{(V)}=\Upsilon \sqrt{c^{(V)} c_{c}^{(V)}\left(1-c_{c}^{(V)}\right)}\left(e^{\frac{\eta}{2}}-e^{-\frac{\eta}{2}}\right), \\
& \eta=\phi_{c}^{(V)}-\phi^{(V)} .
\end{aligned}
$$

The asymptotic solution can then be found by expanding as follows

$$
\begin{array}{lll}
c^{(V)}=c_{0}^{(V)}(t)+\cdots, & \mathcal{F}^{(V)}=\mathcal{F}_{0}^{(V)}(t)+\cdots, \\
j^{(V)}=j_{0}^{(V)}(t)+\cdots, & & \phi^{(V)}=\Theta^{-1} \phi_{-1}^{(V)}(t)+\phi_{0}^{(V)}(x, t)+\cdots, \\
j_{c}^{(V)}=I(t)-j_{0}^{(V)}(t)+\cdots, & \phi_{c}^{(V)}=\Theta^{-1} \phi_{-1}^{(V)}(t)+\phi_{c, 0}^{(V)}(x, t)+\cdots, \\
c_{c}^{(V)}=c_{c, 0}^{(V)}(t)+\cdots & & G^{(V)}=G_{0}^{(V)}(x, t)+\cdots,
\end{array}
$$

which, by design, satisfy equations (177)-(185). The $O(1)$ variables for the ionic and electronic potentials $\phi_{0}^{(V)}$ and $\phi_{c, 0}^{(V)}$ are to be solved. A closed equation for these variables can be 
found by substituting equations (180) and (182) into equations (179) and (181) respectively, adding the two together and substituting the expansions into the resulting equation giving:

$$
\frac{\partial^{2}}{\partial v^{2}}\left(\phi_{c, 0}^{(V)}-\phi_{0}^{(V)}\right)=\bar{K}(t) \sinh \left(\frac{\phi_{c, 0}^{(V)}-\phi_{0}^{(V)}}{2}\right)
$$

where

$$
\bar{K}(t)=2 \Upsilon \frac{\mathcal{B}(x) \kappa\left(c_{0}^{(V)}(t)\right)+\hat{\mathcal{P}}}{\mathcal{B}(x) \kappa\left(c_{0}^{(V)}(t)\right)}\left(c_{0}^{(V)}(t)\right)^{1 / 2}\left(c_{c, 0}^{(V)}(t)\right)^{1 / 2}\left(1-c_{c, 0}^{(V)}(t)\right)^{1 / 2} .
$$

An analytical solution to equation (187)

$$
\phi_{c, 0}^{(V)}-\phi_{0}^{(V)}=\bar{\phi}(t) \pm 4 \log \left(\operatorname{coth}\left(\sqrt{\frac{\bar{K}(t)}{4}}\left(v_{0}(t)-v\right)\right)\right)
$$

where $v_{0}(t)$ and $\bar{\phi}(t)$ are constants of integration, which will be determined by matching to the adjacent regions. Performing this matching will require the limiting behaviours of this solution, which are

$$
\begin{aligned}
& \phi_{c, 0}^{(V)}-\phi_{0}^{(V)} \rightarrow \bar{\phi}(t) \quad \text { as } v \rightarrow-\infty, \\
& \phi_{c, 0}^{(V)}-\phi_{0}^{(V)} \sim 4 \log \left(\sqrt{\frac{\bar{K}(t)}{4}}\left(v_{0}-v\right)\right) \quad \text { as } v \nearrow v_{0} .
\end{aligned}
$$

Region $(V I)$ : right-hand main reaction region The right-hand reaction region, located about $x=s_{2}(t)$, has width $O(\Theta)$ and is therefore studied under a change of coordinates given by

$$
v_{0}-v=\Theta w
$$

and so the local equations become

$$
\begin{gathered}
\mathcal{N}\left(-\Theta^{-1} \dot{s}_{2}(t) \frac{\partial c^{(V I)}}{\partial w}+\frac{\partial c^{(V I)}}{\partial t}\right)+\Theta^{-1} \frac{\partial \mathcal{F}^{(V I)}}{\partial w}=0, \\
\mathcal{F}^{(V I)}=-\Theta^{-1} \mathcal{B}(x) D\left(c^{(V I)}\right) \frac{\partial c^{(V I)}}{\partial w}-\Gamma\left(1-t^{+}\right) j^{(V I)} \\
\Theta^{-1} \frac{\partial j^{(V I)}}{\partial w}=G^{(V I)}, \\
j^{(V I)}=-\frac{\mathcal{B}(x) \kappa\left(c^{(V I)}\right)}{\hat{\mathcal{P}}}\left(\frac{\partial \phi^{(V I)}}{\partial w}-2 \frac{1-t^{+}}{c^{(V I)}} \frac{\partial c^{(V I)}}{\partial w}\right), \\
\Theta^{-1} \frac{\partial j_{c}^{(V I)}}{\partial w}=-G^{(V I)},
\end{gathered}
$$




$$
\begin{gathered}
j_{c}^{(V I)}=-\frac{\partial \phi_{c}^{(V I)}}{\partial w}, \\
-\Theta^{-1} \dot{s}_{2}(t) \frac{\partial c_{c}^{(V I)}}{\partial w}+\frac{\partial c_{c}^{(V I)}}{\partial t}=-G^{(V I)}, \\
G^{(V I)}=\Upsilon \sqrt{c^{(V I)} c_{c}^{(V I)}\left(1-c_{c}^{(V I)}\right)}\left(e^{\frac{\eta}{2}}-e^{-\frac{\eta}{2}}\right), \\
\eta=\phi_{c}^{(V I)}-\phi^{(V I)} .
\end{gathered}
$$

The asymptotic solution can then be found by expanding as follows

$$
\begin{array}{lll}
c^{(V I)}=c_{0}^{(V I)}(t)+\cdots, & \mathcal{F}^{(V I)}=\mathcal{F}_{0}^{(V I)}(t)+\cdots, \\
j^{(V I)}=j_{0}^{(V I)}(x, t)+\cdots, & \phi^{(V I)}=\Theta^{-1} \phi_{-1}^{(V I)}(t)+\phi_{0}^{(V I)}(x, t)+\cdots, \\
j_{c}^{(V I)}=I(t)-j_{0}^{(V I)}(x, t)+\cdots, & \phi_{c}^{(V I)}=\Theta^{-1} \phi_{-1}^{(V I)}(t)-2 \log (\Theta)+\phi_{c, 0}^{(V I)}(x, t)+\cdots, \\
c_{c}^{(V I)}=c_{c, 0}^{(V I)}(x, t)+\cdots, & G^{(V I)}=\Theta^{-1} G_{-1}^{(V I)}(x, t)+\cdots
\end{array}
$$

which, by design, satisfy equations (193)-(194). Upon substituting equation (196) into (195) and substituting equation (198) into (197) we find that, by substitution of the expansions, the first order variables $c_{c, 0}^{(V I)}$ and $j_{0}^{(V I)}$, as well as the second order variables $\phi_{0}^{(V I)}$ and $\phi_{c, 0}^{(V I)}$, satisfy the following equations:

$$
\begin{array}{r}
\frac{\partial^{2}}{\partial w^{2}}\left(\phi_{c, 0}^{(V I)}-\phi_{0}^{(V I)}\right)=\frac{B(x) \kappa\left(c^{(V I)}(t)\right)+\hat{\mathcal{P}}}{\mathcal{B}(x) \kappa\left(c^{(V I)}(t)\right)} G_{-1}^{(V I)}, \\
-\dot{s}_{2}(t) \frac{\partial c_{c, 0}^{(V I)}}{\partial w}=G_{-1}^{(V I)}, \quad \frac{\partial j_{0}^{(V I)}}{\partial w}=-\dot{s}_{2}(t) \frac{\partial c_{c, 0}^{(V I)}}{\partial w} . \\
G_{-1}^{(V I)}=\Upsilon\left(c_{0}^{(V I)}\right)^{1 / 2}\left(c_{c, 0}^{(V I)}\right)^{1 / 2}\left(1-c_{c, 0}^{(V I)}\right)^{1 / 2} \exp \left(\frac{\phi_{c, 0}^{(V I)}-\phi_{0}^{(V I)}}{2}\right) .
\end{array}
$$

The pair of equations for $\phi_{c, 0}^{(V I)}-\phi_{0}^{(V I)}$ and $c_{c, 0}^{(V I)}$ are to be solved subject to matching conditions from the neighbouring regions, namely $(V)$ and $(V I I)$.

$$
\left.\begin{array}{l}
\phi_{c, 0}^{(V I)}-\phi_{0}^{(V I)} \sim 4 \log \left(-\sqrt{\frac{\bar{K}(t)}{4}} w\right), \\
\frac{\partial}{\partial w}\left(\phi_{c, 0}^{(V I)}-\phi_{0}^{(V I)}\right) \sim I(t), \\
c_{c, 0}^{(V I)} \sim c_{c, 0}^{(I V)},
\end{array}\right\} \text { as } w \rightarrow-\infty
$$

The limiting behaviour (191) can be rewritten in the spatial variables of region $(V I)$, see (192), as

$$
2 \log \Theta+O(1) \sim \phi_{c, 0}^{(V I)}-\phi_{0}^{(V I)}
$$

and for matching this requires (206a). Matching the concentration in the solid leads to the condition (206c) whilst the current requires

$$
j_{0}^{(V I)}-j_{c, 0}^{(V I)} \rightarrow-I(t) \text { as } w \rightarrow-\infty
$$

giving (206b). 
Matching regions $(I V)-(V I I)$ across right reaction front near $x=s_{2}(t)$ Upon matching the leading order lithium concentrations and fluxes (in the electrolyte) across the regions adjacent to the right-hand reaction front situated about $x=s_{2}(t)$ we find that

$$
\begin{gathered}
\left.c_{0}^{(I V)}\right|_{x \nearrow s_{2}(t)}=c_{0}^{(V)}(t)=c_{0}^{(V I)}(t)=\left.c_{0}^{(V I I)}\right|_{x \searrow_{2}(t)}, \\
\left.\mathcal{F}_{0}^{(I V)}\right|_{x \nearrow s_{2}(t)}=\mathcal{F}_{0}^{(V)}(t)=\mathcal{F}_{0}^{(V I)}(t)=\left.\mathcal{F}_{0}^{(V I I)}\right|_{x \searrow_{s_{2}}(t) .} .
\end{gathered}
$$

Performing a similar operation for the leading order electric potentials we obtain the following matching conditions:

$$
\left.\phi_{c,-1}^{(I V)}\right|_{x \nearrow s_{2}(t)}(t)=\phi_{c,-1}^{(V)}(t)=\phi_{-1}^{(V I)}(t)=\left.\phi_{-1}^{(V I I)}\right|_{x \searrow s_{2}(t)} .
$$

On matching the leading order electrolyte potentials across regions $(V I)$ and $(V I I)$ we find

$$
\phi_{-1}^{(V I)}(t)=\phi_{-1}^{(V I I)}(t) .
$$

The $O(1)$ terms for the difference between the ionic and electronic potentials must be matched between regions $(V)$ and $(V I)$. This leads to the requirement that

$$
\bar{\phi}(t)=\left.\phi_{c, 0}^{(I V)}\right|_{x \nearrow s_{2}(t)}-\left.\phi_{0}^{(I V)}\right|_{x s_{2}(t)} .
$$

Matching the leading order lithium content inside the electrode particles across all four regions leads to the conditions

$$
\left.c_{c, 0}^{(I V)}\right|_{x \nearrow_{s_{2}(t)}}=c_{c, 0}^{(V)}(t)=\left.c_{c, 0}^{(V I)}\right|_{w \rightarrow-\infty},\left.\quad c_{c, 0}^{(V I)}\right|_{w \rightarrow \infty}=1 .
$$

Finally, the matching conditions on the leading order ionic current densities $j$ are

$$
\left.j_{0}^{(I V)}\right|_{x \nearrow_{2}(t)}=j_{0}^{(V)}(t)=\left.j_{0}^{(V I)}\right|_{w \rightarrow-\infty},\left.\quad j_{0}^{(V I)}\right|_{w \rightarrow \infty}=0 .
$$

The position of the first reaction layer $s_{2}(t)$ is found by integrating and rearranging equation (204.b) and applying conditions (214)-(215), yielding

$$
\dot{s}_{2}(t)=\frac{\left.j_{0}^{(V I)}\right|_{w \rightarrow \infty}-\left.j_{0}^{(V I)}\right|_{w \rightarrow-\infty}}{\left.c_{c, 0}^{(V I)}\right|_{w \rightarrow \infty}-\left.c_{c, 0}^{(V I)}\right|_{w \rightarrow-\infty}}=-\frac{j_{0}^{(I I I)}(t)}{1-c_{c, 0}^{(I I I)}(t)},
$$

with the initial condition $\left.s_{2}(t)\right|_{t=0}=1$.

\section{A.4 Matching the outer regions together}

Following $\S$ A.2 and $\S$ A.3 a closed problem involving only the outer regions, namely $(I),(I V)$, and (VII), can be formed. Taking equations (168)-(169) and (209)-(210) we have that

$$
\begin{gathered}
\left.c_{0}^{(I)}\right|_{x \nearrow s_{1}(t)}=\left.c_{0}^{(I V)}\right|_{x \searrow s_{1}(t)},\left.\quad c_{0}^{(I V)}\right|_{x \nearrow s_{2}(t)}=\left.c_{0}^{(V I I)}\right|_{x \searrow s_{2}(t)}, \\
\left.\mathcal{F}_{0}^{(I)}\right|_{x \nearrow s_{1}(t)}=\left.\mathcal{F}_{0}^{(I V)}\right|_{x \searrow_{\searrow}(t)},\left.\quad \mathcal{F}_{0}^{(I V)}\right|_{x \nearrow s_{2}(t)}=\left.\mathcal{F}_{0}^{(V I I)}\right|_{x \searrow s_{2}(t)} .
\end{gathered}
$$

which translate to imposing continuity of concentration and flux of the anions in the electrolyte across the narrow reaction fronts. The current densities can be determined as known functions of the anion concentration $c$ at leading order in the outer regions. This is achieved by taking equations (106a), (113a), and (126), giving

$$
j^{(I)}=I(t), \quad j^{(I V)}=I(t) \frac{\mathcal{B}(x) \kappa\left(c_{0}^{(I V)}\right)}{\mathcal{B}(x) \kappa\left(c_{0}^{(I V)}\right)+\hat{\mathcal{P}}}, \quad j^{(V I I)}=0 .
$$




\section{B Analytical expressions for the electrode and elec- trolyte properties}

The equilibrium potential for LFP shown in figure 2 panel (c) is given by [49].

$$
\begin{aligned}
U_{c}^{*}\left(c_{c}^{*}\right)=3.114559+4.438792 \arctan & \left.-71.7352 \frac{c_{c}^{*}}{\hat{c}_{c}}+70.85337\right) \\
& -4.240252 \arctan \left(-68.5605 \frac{c_{c}^{*}}{\hat{c}_{c}}+67.730082\right) \mathrm{V}
\end{aligned}
$$

The cell is filled with 1M LiPF 6 in 1:1 EC:DMC electrolyte. For the ionic diffusivity and conductivity of anions in the electrolyte we use [53]

$$
\begin{aligned}
& D^{*}\left(c^{*}\right)=5.253 \times 10^{-10} \exp \left(-3.071 \times 10^{-4} c^{*}\right) m^{2} s^{-1}, \\
& \kappa^{*}\left(c^{*}\right)=10^{-4} c^{*}\left(5.2069096-0.002143638 c^{*}+2.34402 \times 10^{-7} c^{* 2}\right) S m^{-1} \text {, }
\end{aligned}
$$

and for the ionic permeability we use a Bruggeman approximation for the electrolyte inside the cathode and a specify a value for that in the separator $[6,16]$

$$
\mathcal{B}^{*}\left(x^{*}\right)= \begin{cases}0.55 & x^{*}<0 \\ \epsilon_{l}^{1.5} & x^{*} \geq 0\end{cases}
$$

where $\epsilon_{l}$ is the local volume fraction of electrolyte. 